\title{
Finite-Time Control for Attitude Tracking Maneuver of Rigid Satellite
}

\author{
Mingyi Huo, ${ }^{1}$ Xing Huo, ${ }^{2}$ Hamid Reza Karimi, ${ }^{3}$ and Jianfei $\mathrm{Ni}^{2}$ \\ ${ }^{1}$ Department of Control Science and Engineering, Harbin Institute of Technology, Harbin 150001, China \\ ${ }^{2}$ College of Engineering, Bohai University, Jinzhou 121000, China \\ ${ }^{3}$ Department of Engineering, Faculty of Engineering and Science, The University of Agder, 4898 Grimstad, Norway
}

Correspondence should be addressed to Xing Huo; hmyi888@163.com

Received 7 November 2013; Accepted 8 January 2014; Published 26 February 2014

Academic Editor: Junmin Wang

Copyright (C) 2014 Mingyi Huo et al. This is an open access article distributed under the Creative Commons Attribution License, which permits unrestricted use, distribution, and reproduction in any medium, provided the original work is properly cited.

\begin{abstract}
The problem of finite-time control for attitude tracking maneuver of a rigid spacecraft is investigated. External disturbance, unknown inertia parameters are addressed. As stepping stone, a sliding mode controller is designed. It requires the upper bound of the lumped uncertainty including disturbance and inertia matrix. However, this upper bound may not be easily obtained. Therefore, an adaptive sliding mode control law is then proposed to release that drawback. Adaptive technique is applied to estimate that bound. It is proved that the closed-loop attitude tracking system is finite-time stable. The tracking errors of the attitude and the angular velocity are asymptotically stabilized. Moreover, the upper bound on the lumped uncertainty can be exactly estimated in finite time. The attitude tracking performance with application of the control scheme is evaluated through a numerical example.
\end{abstract}

\section{Introduction}

Satellites need to perform attitude tracking maneuvers to accomplish orbital missions. For example, satellite surveillance and communication often have need of highly accurate slewing and/or pointing maneuvers that require the satellite to rotate along a relatively large-angle amplitude trajectory. As discussed in [1], these requirements necessitate the use of nonlinear differential equations for the kinematics and dynamics in the attitude control system. The attitude tracking problem is further complicated by the uncertainty of the satellite mass and inertia properties due to onboard payload motion, rotation of solar arrays, and fuel consumption. Addressing these issues has attracted considerable interest in the existing literature. A number of control design approaches by using adaptive control [2-4], $\mathscr{H}_{\infty}[5,6]$, Backstepping control [7], robust control [8,9], and optimal control [10, 11] have been developed. However, there still remain open problems in this field that are of great theoretical and practical interest. For example, there currently exist few unified frameworks for the design of simple control structures to reject external disturbance.
Several solutions to the attitude control problem with disturbance rejection have been presented $[12,13]$. In [14], a general trajectory tracking design methodology was developed for a flexible spacecraft subject to a gravity-gradient disturbance. Treating the flexible dynamics as an additional disturbance, an attitude controller was synthesized by using adaptive control. A similar problem was considered in [15] for three-axis-controller satellites with flexible appendages. A disturbance compensator was developed to accommodate external disturbances. In [16], a continuous globally stable tracking control was presented. Control input saturation and external disturbances were considered simultaneously, and the knowledge of inertia parameters was not needed to implement the controller. In [17], an attitude tracking controller was proposed to guarantee global asymptotic stability of the system in the presence of disturbances and parameter uncertainties. Although asymptotic rejection of external disturbances was achieved in [18], the technique was developed for a particular type of disturbance composed of sinusoidal functions with arbitrarily unknown amplitude and phase angles, but known frequencies. An inertia-free attitude tracking control was synthesized for a rigid spacecraft 
[19]. The proposed controller addressed the problem of disturbance rejection. However, the control law could only guarantee almost global stability of the closed-loop system. In [20], the attitude regulation control problem for flexible spacecraft was investigated. The external disturbances and model uncertainties were handled by using passivity-based control technique.

The sliding mode control (SMC) is a powerful theory for controlling uncertain systems [21]. The main advantages are that the SMC system has great robustness with respect to uncertain parameters and external disturbances. Hence, applying SMC to design attitude control for satellite has been intensively carried out [22]. The first attempt using SMC to achieve large angle attitude maneuver for spacecraft was made in [23] and further pursued in [24]. A higher-order SMC control scheme was presented in [25]. The problem of spacecraft attitude tracking maneuvers was addressed. External disturbances and uncertain inertia parameters were explicitly addressed. To reduce the effect of external disturbance on the attitude control performance, an observer was proposed in [26] to estimate the disturbance. Using the estimated value, a sliding mode attitude controller was synthesized. In [27], an SMC-based control algorithm was designed for rigid spacecraft to perform attitude stabilization maneuver. External disturbance and inertia uncertainty were investigated and handled by designing adaptive law. In [28], adaptive law was synthesized to estimate the disturbance; sliding mode controllers were designed to achieve attitude tracking of a rigid spacecraft subject to uncertain inertia and external disturbance. The problem of attitude tracking control in the presence of uncertain inertia and external disturbances was further studied in $[29,30]$.

When applying SMC to design attitude controller, it usually consists of two stages: (i) choosing a stable manifold as the sliding surface and (ii) designing controller to drive the system reaching the sliding surface. The attitude tracking problem in the preceding SMC-based studies is solved in the framework of traditional SMC. A linear sliding mode is utilized to describe the desired performance of the closed-loop systems; that is, the system state variables slide to the equilibrium point exponentially on the sliding surface. Although the convergence rate may be arbitrarily fast from adjusting appropriate parameters, stabilizing dynamical systems cannot be achieved in finite time. Obviously, the infinite-settling time criterion is not an option during critical phases of some high demanding real-time missions. Accomplishing finite-time error convergence is more desirable in practice. Therefore, the design of finite-time control for satellite attitude system has been investigated recently $[31,32]$.

To achieve finite-time convergence of dynamical systems, terminal SMC (TSMC) has been a widely used approach $[33,34]$. For example, a terminal SMC is applied to design attitude tracking control in [30]. Finite-time reachability of a given desired attitude motion was guaranteed. Taking external disturbances into account, an adaptive TSMC law was designed to achieve finite-time attitude tracking [35]. In [36], the finite-time attitude stabilization problem was studied by using TSMC. However, the states of attitude control system only converged to a small region of the

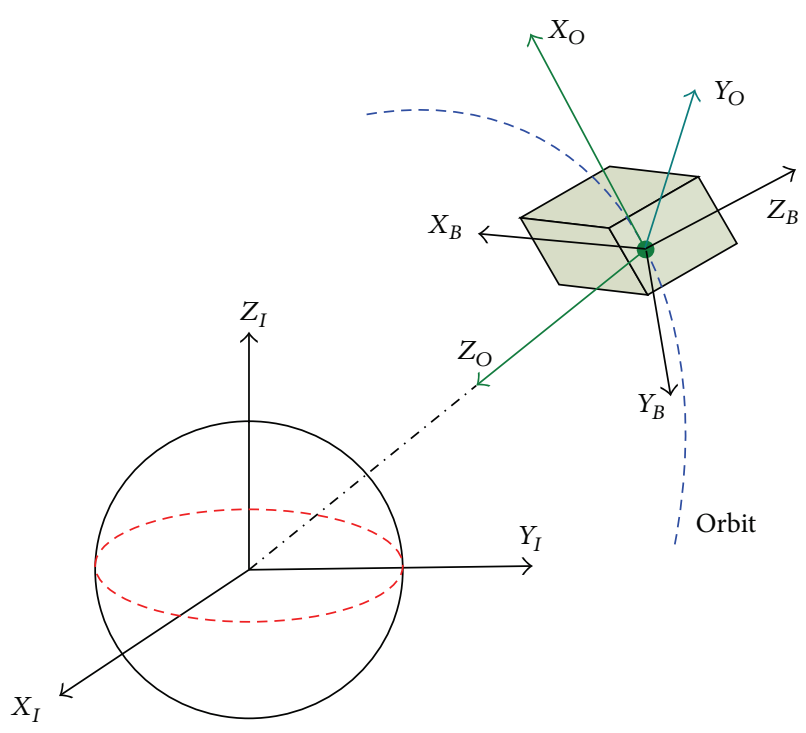

FIgURE 1: Definition of the coordinate reference frames.

origin in the presence of external disturbance. Although the attitude stabilization was achieved for a rigid spacecraft subject to disturbances and uncertain inertia by using TSMC, the attitude was only stabilized to a small region instead of zero in finite time [37]. In [38], the authors focused on the problem of rapid reorienting of a spacecraft with external disturbance and uncertain nature of the dynamics. In [39], Chebyshev neural network was applied to estimate disturbance and system uncertainty of spacecraft attitude system. The controller designed by using TSMC achieved finite-time tracking. However, it was not able to stabilize the attitude tracking error to zero. In [40], TSMC was applied to achieve satellites formation flying. More recently, a fast TSMC scheme was proposed to accomplish attitude tracking maneuver with finite-time convergence [41].

Although many SMC or TSMC based attitude tracking control schemes have been developed with external disturbances and uncertain inertia parameters investigated, most of them include two drawbacks. (1) The attitude tracking error was only stabilized to a small region containing the origin; asymptotical stability was not guaranteed, although finite-time convergence was achieved. (2) To handle external disturbance and uncertain inertia matrix, adaptive control technique was applied to estimate the upper bound on those two items. However, asymptotical convergence of the estimation was not achieved. With a view to tackle those two issues, this work investigates the feasibility of finite-time tracking of desired attitude trajectory in the presence external disturbances and uncertain inertia parameters. An adaptive SMC control approach is presented. The main contributions of this work are that those stated two challenges are addressed.

The remainder of this paper is organized as follows. Section 2 contains the derivation of the satellite attitude tracking model and the control problem formulation. The finite-time attitude tracking controller is presented in Section 3. In Section 4, simulation results with the application 


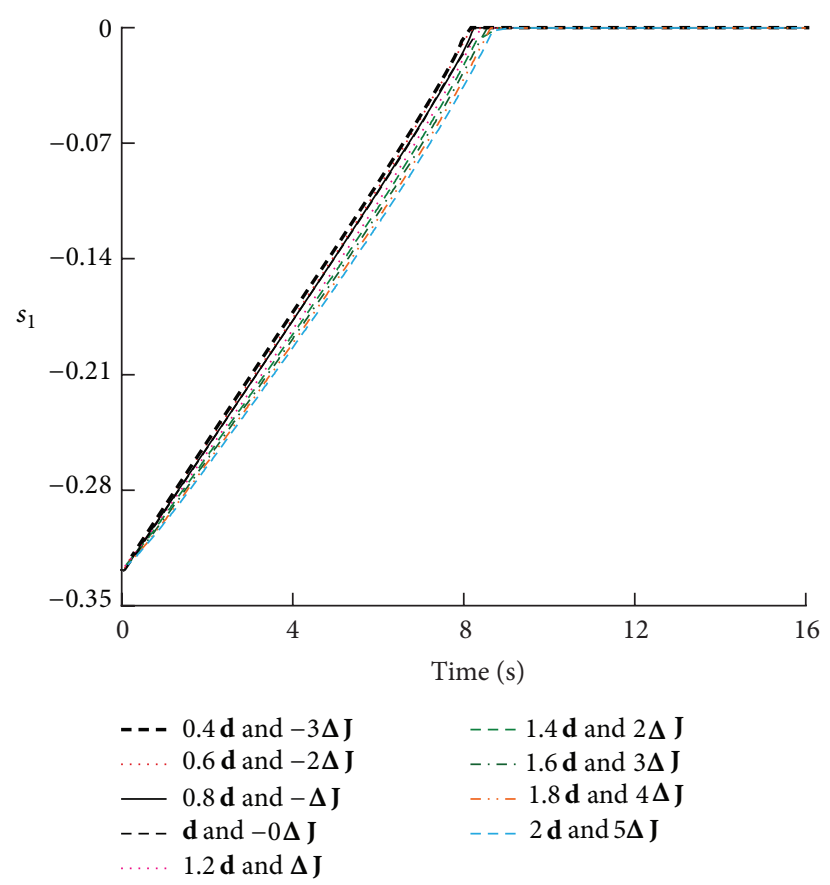

(a) The whole response

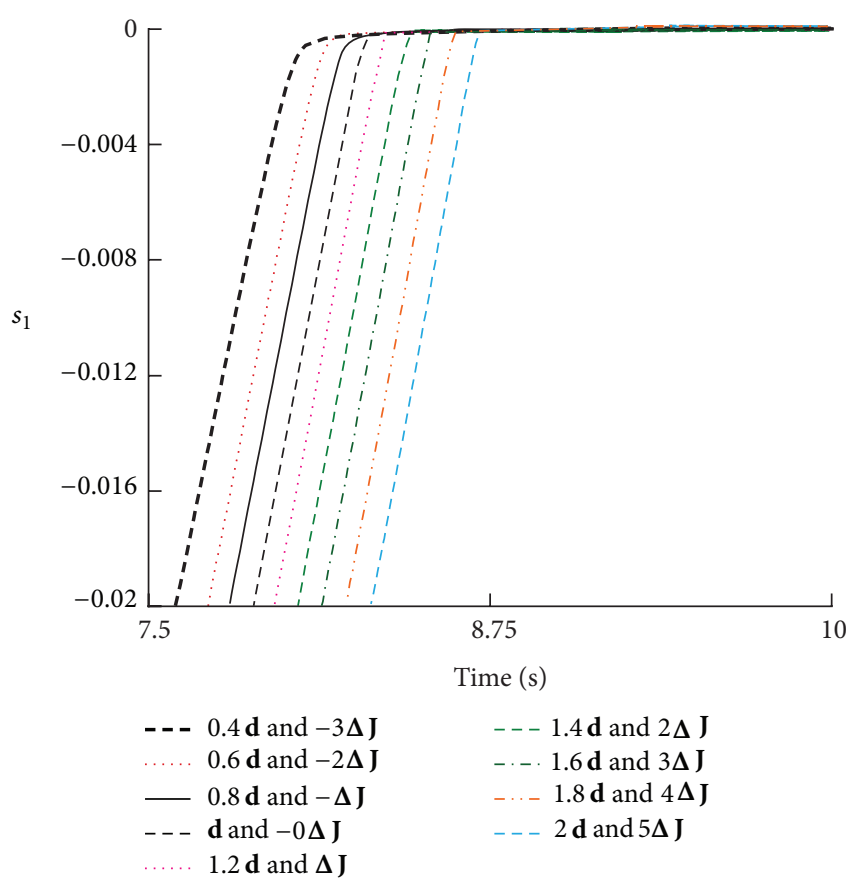

(b) The steady-state behavior

Figure 2: The sliding surface $s_{1}$ with different disturbance and uncertain inertia.

of the derived control scheme to a rigid satellite are presented. Section 5 presents some concluding remarks and future work.

\section{System Description and Problem Formulation}

Throughout the paper, $\mathbf{I}_{n}$ denotes a $n \times n$ unit matrix, and $\|\cdot\|$ denotes the Euclidean norm or its induced norm. For vector $\mathbf{x}=\left[\begin{array}{llll}x_{1} & x_{2} & \cdots & x_{n}\end{array}\right]^{\mathrm{T}} \in \mathfrak{R}^{n}$ and positive scalar $m$, a diagnosis matrix is defined as $\left|\boldsymbol{\sigma}_{e}\right|^{\gamma}=\operatorname{diag}\left(\left[\begin{array}{llll}\left|x_{1}\right|^{\gamma} & \left|x_{2}\right|^{\gamma} & \cdots & \left|x_{n}\right|^{\gamma}\end{array}\right]^{\mathrm{T}}\right)$, and a vector $\operatorname{sgn}(\mathbf{x})=\left[\begin{array}{llll}\operatorname{sign}\left(x_{1}\right) & \operatorname{sign}\left(x_{2}\right) & \cdots & \operatorname{sign}\left(x_{n}\right)\end{array}\right]^{\mathrm{T}} \epsilon$ $\mathfrak{R}^{n}$ is also defined with sign( $(\cdot)$ denoting the sign function.

2.1. Satellite Dynamics. Consider a rigid satellite moving in a circular orbit. The coordinate systems used in the attitude control are shown in Figure 1. The inertial frame $\mathscr{I}\left(X_{I}\right.$, $\left.Y_{I}, Z_{I}\right)$ with its origin at the center of the Earth is used to determine the orbital position of the satellite. The orbit reference frame $\mathcal{O}\left(X_{O}, Y_{O}, Z_{O}\right)$, rotating about the $Y_{O}$ axis with respect to $\mathscr{I}$, has its origin located in the mass center of the satellite. The axes of $\mathcal{O}$ are chosen such that the roll axis $X_{O}$ is in the flight direction, the pitch axis $Y_{O}$ is perpendicular to the orbital plane, and the yaw axis $Z_{O}$ points toward the Earth. The last reference system used is the body-fixed frame $\mathscr{B}\left(X_{B}, Y_{B}, Z_{B}\right)$. It has the same origin as $\mathcal{O}$, and its axes coincide with the principal axis of inertia.

With the coordinate references defined in Figure 1, the dynamic model of a rigid satellite can be expressed as [42]

$$
\dot{\boldsymbol{\sigma}}=\frac{1}{4}\left[\left(1-\boldsymbol{\sigma}^{\mathrm{T}} \boldsymbol{\sigma}\right) \mathbf{I}_{3}+2 \boldsymbol{\sigma}^{\times}+2 \boldsymbol{\sigma} \boldsymbol{\sigma}^{\mathrm{T}}\right] \boldsymbol{\omega}=\mathbf{G}(\boldsymbol{\sigma}) \boldsymbol{\omega},
$$

$$
\mathrm{J} \dot{\boldsymbol{\omega}}=-\boldsymbol{\omega}^{\times} \mathrm{J} \boldsymbol{\omega}+\mathbf{u}+\mathbf{d},
$$

where $\mathbf{J} \in \mathfrak{R}^{3 \times 3}$ is the positive-definite, symmetric inertia matrix, $\boldsymbol{\omega} \in \mathfrak{R}^{3}$ is the angular velocity of the body-fixed reference frame $\mathscr{B}$ with respect to the inertial reference frame $\mathscr{I}$ and expressed in $\mathscr{B}, \mathbf{u} \in \mathfrak{R}^{3}$ is a vector of control torques, d $\in \mathfrak{R}^{3}$ is a vector of external disturbance, and the notation $\mathbf{x}^{\times}, \forall \mathbf{x}=\left[\begin{array}{lll}x_{1} & x_{2} & x_{3}\end{array}\right]^{\mathrm{T}}$, denotes the following cross-product matrix:

$$
\mathbf{x}^{\times}=\left[\begin{array}{ccc}
0 & -x_{3} & x_{2} \\
x_{3} & 0 & -x_{1} \\
-x_{2} & x_{1} & 0
\end{array}\right]
$$

In (1), $\boldsymbol{\sigma}=\left[\begin{array}{lll}\sigma_{1} & \sigma_{2} & \sigma_{3}\end{array}\right]^{\mathrm{T}} \in \mathfrak{R}^{3}$ represents the modified Rodriguez parameters describing the orientation of the bodyfixed frame $\mathscr{B}$ with respect to the inertial reference frame $\mathscr{I}$, which is defined by [42]

$$
\boldsymbol{\sigma}=\mathbf{e} \tan \left(\frac{\zeta(t)}{4}\right), \quad 0^{\circ} \leq \zeta(t)<360^{\circ}
$$

with $\mathbf{e} \in \mathfrak{R}^{3}$ and $\zeta \in \mathfrak{R}$ denoting the Euler eigenaxis and eigenangle, respectively. The attitude description using the modified Rodriguez parameters has an advantage of being valid for eigenaxis rotations up to 360 degrees. The rotation matrix that brings $\mathscr{I}$ onto $\mathscr{B}$, denoted by $\mathbf{R}(\boldsymbol{\sigma}) \in \mathfrak{R}^{3 \times 3}$, is defined as follows:

$$
\mathbf{R}(\boldsymbol{\sigma}) \triangleq \mathbf{I}_{3}-\frac{4\left(1-\boldsymbol{\sigma}^{\mathrm{T}} \boldsymbol{\sigma}\right)}{\left(1+\boldsymbol{\sigma}^{\mathrm{T}} \boldsymbol{\sigma}\right)^{2}} \boldsymbol{\sigma}^{\times}+\frac{8 \boldsymbol{\sigma}^{\times} \boldsymbol{\sigma}^{\times}}{\left(1+\boldsymbol{\sigma}^{\mathrm{T}} \boldsymbol{\sigma}\right)^{2}} .
$$




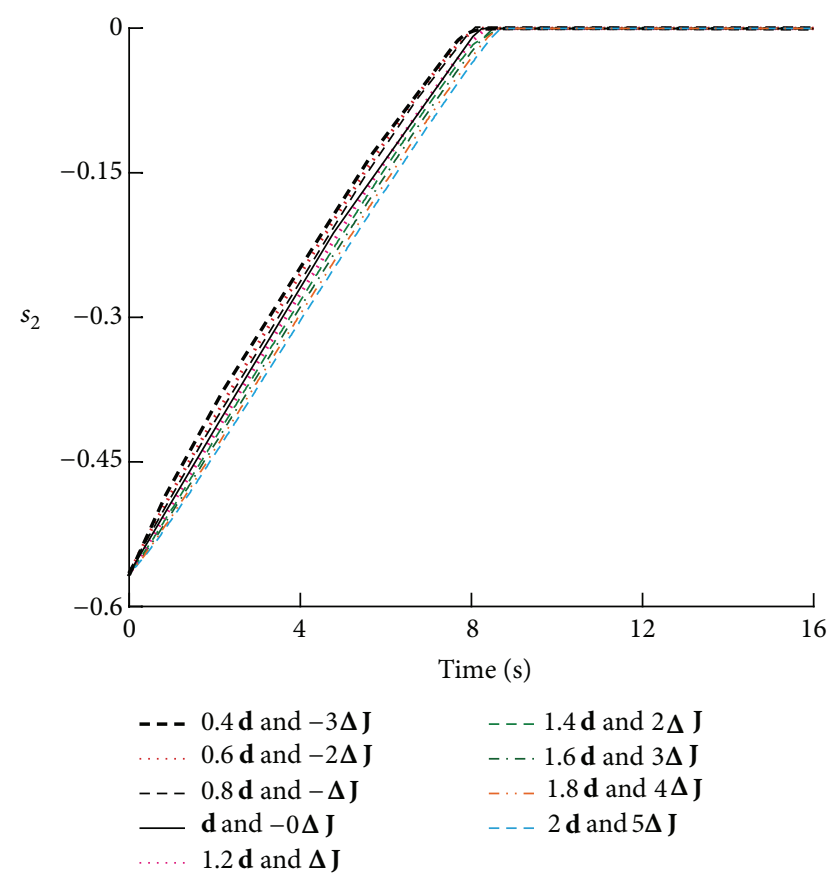

(a) The whole response

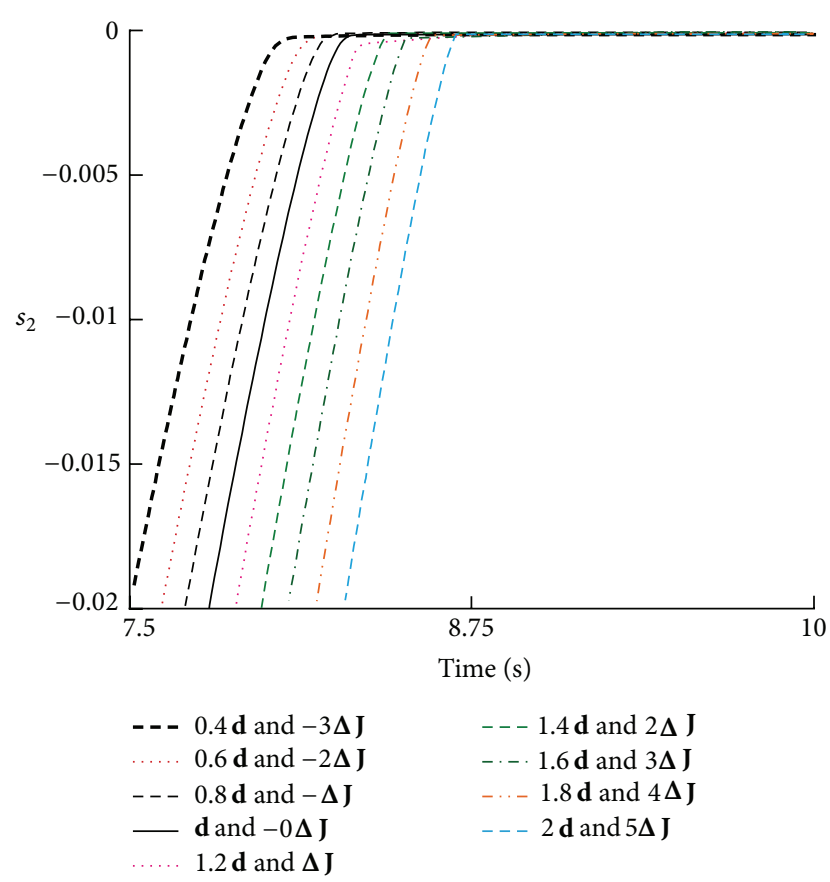

(b) The steady-state behavior

FIgURE 3: The sliding surface $s_{2}$ with different disturbance and uncertain inertia.

2.2. Open-Loop Attitude Tracking Error Dynamics. Assume that the desired attitude of the satellite can be described by a desired, body-fixed reference frame $\mathscr{D}$ whose orientation with respect to $\mathscr{I}$ is specified by the desired modified Rodriguez parameters $\sigma_{d} \in \mathfrak{R}^{3}$. The desired angular velocity of $\mathscr{D}$, with respect to $\mathscr{I}$ expressed in $\mathscr{D}$, is denoted by $\omega_{d} \in$ $\mathfrak{R}^{3}$. The attitude tracking error $\boldsymbol{\sigma}_{e}=\left[\begin{array}{lll}\sigma_{e 1} & \sigma_{e 2} & \sigma_{e 3}\end{array}\right]^{\mathrm{T}} \in \mathfrak{R}^{3}$ between the actual attitude $\sigma$ and the desired attitude $\sigma_{d}$ is defined as

$$
\boldsymbol{\sigma}_{e}=\frac{\left(1-\boldsymbol{\sigma}_{d}^{\mathrm{T}} \boldsymbol{\sigma}_{d}\right) \boldsymbol{\sigma}-\left(1-\boldsymbol{\sigma}^{\mathrm{T}} \boldsymbol{\sigma}\right) \boldsymbol{\sigma}_{d}+2 \boldsymbol{\sigma}^{\times} \boldsymbol{\sigma}_{d}}{1+\boldsymbol{\sigma}^{\mathrm{T}} \boldsymbol{\sigma} \boldsymbol{\sigma}_{d}^{\mathrm{T}} \boldsymbol{\sigma}_{d}+2 \boldsymbol{\sigma}_{d}^{\mathrm{T}} \boldsymbol{\sigma}}
$$

To quantify the mismatch between the actual and desired satellite attitudes, we define the rotation matrix $\mathbf{R}\left(\boldsymbol{\sigma}_{e}\right) \in \mathfrak{R}^{3 \times 3}$ that brings $\mathscr{D}$ onto $\mathscr{B}$ as $\mathbf{R}\left(\boldsymbol{\sigma}_{e}\right)=\mathbf{R}(\boldsymbol{\sigma}) \mathbf{R}\left(\boldsymbol{\sigma}_{d}\right)^{\mathrm{T}}$. Based on the preceding tracking error formulation, we define the angular velocity of $\mathscr{B}$ with respect to $\mathscr{D}$ expressed in $\mathscr{B}$, denoted by $\boldsymbol{\omega}_{e}=\left[\begin{array}{lll}\omega_{e 1} & \omega_{e 2} & \omega_{e 3}\end{array}\right]^{\mathrm{T}} \in \mathfrak{R}^{3}$,

$$
\boldsymbol{\omega}_{e}=\boldsymbol{\omega}-\mathbf{R}\left(\boldsymbol{\sigma}_{e}\right) \boldsymbol{\omega}_{d} .
$$

We can now use (1)-(2) and (6)-(7) to compute the governing nonlinear equations for the open-loop tracking error dynamics as

$$
\begin{gathered}
\dot{\boldsymbol{\sigma}}_{e}=\mathbf{G}\left(\boldsymbol{\sigma}_{e}\right) \boldsymbol{\omega}_{e}, \\
\mathbf{J} \dot{\boldsymbol{\omega}}_{e}=-\left[\boldsymbol{\omega}_{e}+\mathbf{R}\left(\boldsymbol{\sigma}_{e}\right) \boldsymbol{\omega}_{d}\right]^{\times} \mathbf{J}\left(\boldsymbol{\omega}_{e}+\mathbf{R}\left(\boldsymbol{\sigma}_{e}\right) \boldsymbol{\omega}_{d}\right) \\
+\mathbf{J}\left[\boldsymbol{\omega}_{e}^{\times} \mathbf{R}\left(\boldsymbol{\sigma}_{e}\right) \boldsymbol{\omega}_{d}-\mathbf{R}\left(\boldsymbol{\sigma}_{e}\right) \dot{\boldsymbol{\omega}}_{d}\right]+\mathbf{u}+\mathbf{d} .
\end{gathered}
$$

In (8), the matrix $\mathbf{G}\left(\boldsymbol{\sigma}_{e}\right) \in \mathfrak{R}^{3 \times 3}$ in (1) is subject to

$$
\begin{gathered}
\mathbf{G}^{\mathrm{T}}\left(\boldsymbol{\sigma}_{e}\right) \mathbf{G}\left(\boldsymbol{\sigma}_{e}\right)=\left(\frac{1+\boldsymbol{\sigma}_{e}^{\mathrm{T}} \boldsymbol{\sigma}_{e}}{4}\right)^{2} \mathbf{I}_{3}, \\
\mathbf{G}^{-1}\left(\boldsymbol{\sigma}_{e}\right)=\frac{16}{\left(1+\boldsymbol{\sigma}_{e}^{\mathrm{T}} \boldsymbol{\sigma}_{e}\right)^{2}} \mathbf{G}^{\mathrm{T}}\left(\boldsymbol{\sigma}_{e}\right) .
\end{gathered}
$$

During attitude maneuver, the mass property of the satellite may be uncertain or may change due to fuel consumption or payload motion. It thus leads to the uncertain and even time-varying inertia matrix. Consequently, the inertia matrix $\mathbf{J}$ can be denoted as $\mathbf{J}=\mathbf{J}_{0}+\Delta \mathbf{J}(t)$ with $\mathbf{J}_{0} \in \Re^{3 \times 3}$ being the nominal and constant inertia matrix and $\Delta \mathrm{J}(t) \in \mathfrak{R}^{3 \times 3}$ the uncertain inertia. It should be stressed that although $\mathbf{J}$ is uncertain, it remains positive-definite.

2.3. Control Problem Formulation. Given any initial attitude and angular velocity, the control objective to be achieved can be stated as follows. Consider the rigid satellite attitude dynamics described by (1)-(2) in the presence of unknown external disturbance and uncertain inertia parameters; design a control law $\mathbf{u}$ to guarantee that the attitude $\boldsymbol{\sigma}$ can follow that desired attitude $\sigma_{d}$ in finite time. That is, there exists a finite-time $t_{f}$ such that the attitude tracking error $\boldsymbol{\sigma}_{e}(t)=\mathbf{0}$ and the angular velocity $\boldsymbol{\omega}_{e}(t)=\mathbf{0}, t \geq t_{f}$.

\section{Finite-Time Attitude Tracking Control Design}

Because the sliding mode control system has great robustness with respect to both parameter uncertainties and external 


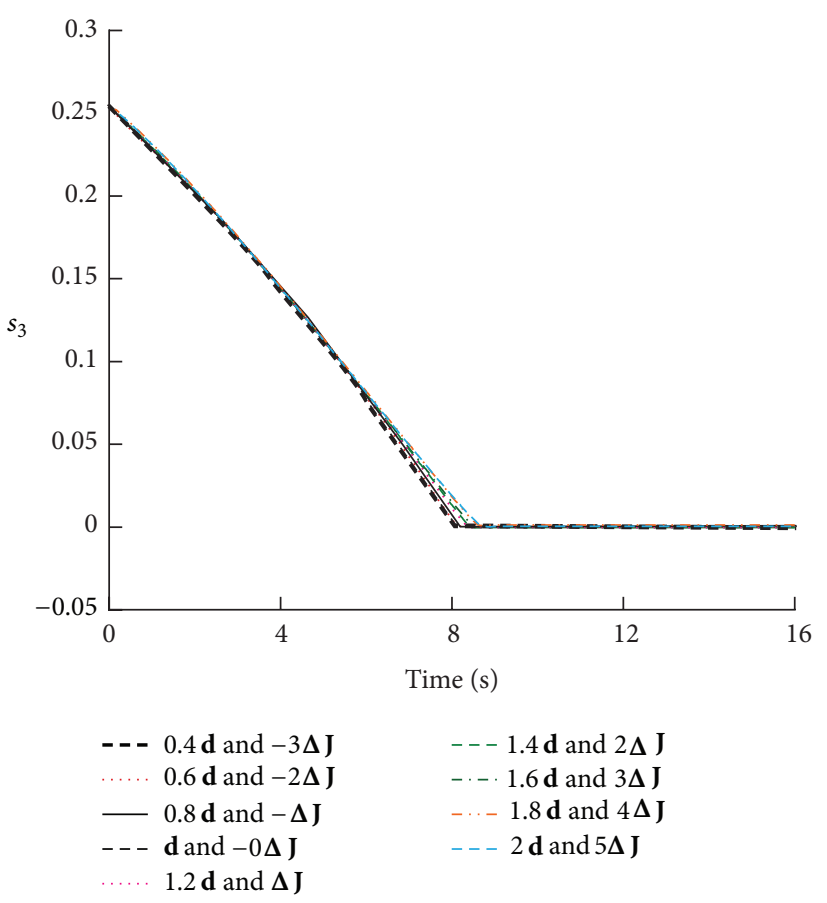

(a) The whole response

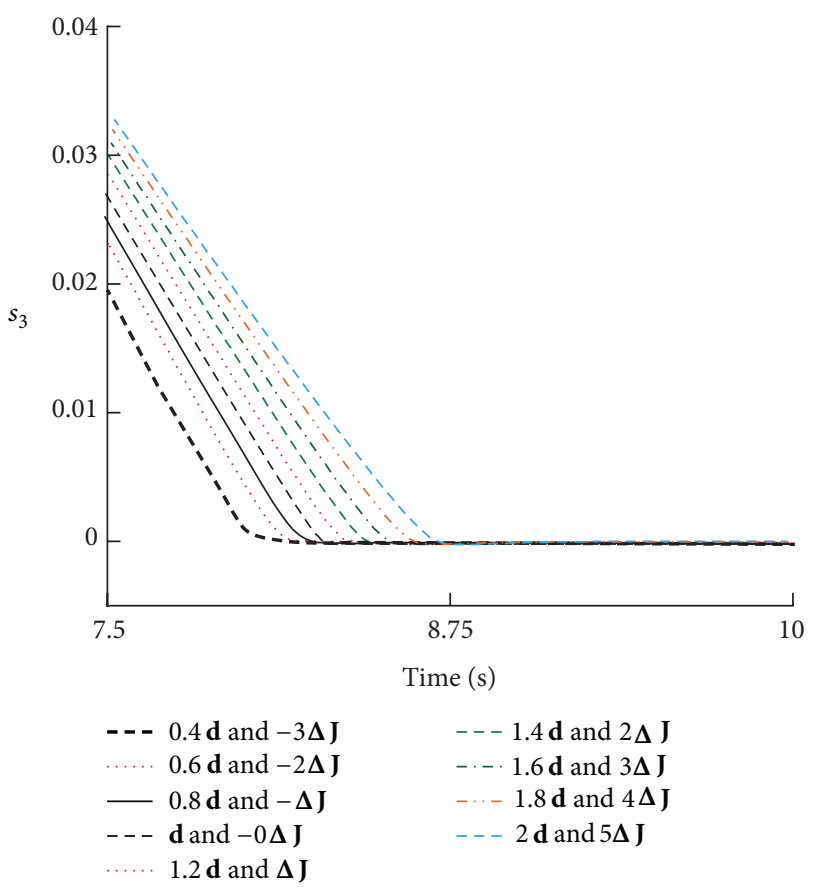

(b) The steady-state behavior

FIgURE 4: The sliding surface $s_{3}$ with different disturbance and uncertain inertia.

disturbance, SMC is adopted to design control law to accomplish the attitude tracking maneuver in finite time. Before giving the control design, one definition and two lemmas are firstly presented which will be utilized in the subsequent control development and analysis.

Definition 1 (see [43]). Consider the following dynamic system:

$$
\dot{\mathbf{x}}(t)=\mathbf{f}(\mathbf{x}(t), t),
$$

where $\mathbf{x}(t) \in \mathfrak{R}^{n}$ is the system state. If there exists a constant $T>0$ (T may depend on the initial state $\mathbf{x}(0)$ ), such that $\lim _{t \rightarrow T}\|\mathbf{x}(t)\|=0$ and $\|\mathbf{x}(t)\|=0$ if $t \geq T$, then the system (11) is finite-time stable.

Lemma 2 (see [43]). Suppose that a continuous, positivedefinite function $V(t)$ satisfies the following differential inequality:

$$
\dot{V}(t) \leq-\alpha V^{\gamma}(t), \quad \forall t \geq t_{0}, V\left(t_{0}\right) \geq 0,
$$

where $\alpha>0$ and $0<\gamma<1$ are two scalars. Then, for any given $t_{0}, V(t)$ satisfies the following inequality:

$$
V^{1-\gamma}(t) \leq V^{1-\gamma}\left(t_{0}\right)-\alpha(1-\gamma)\left(t-t_{0}\right), \quad t_{0} \leq t \leq t_{1},
$$

and $V(t) \equiv 0$ for $\forall t \geq t_{1}$, with $t_{1}$ given by

$$
t_{1}=t_{0}+\frac{V^{1-\gamma}\left(t_{0}\right)}{\alpha(1-\gamma)}
$$

Lemma 3 (see [37]). For any vector $\mathbf{x}=\left[\begin{array}{lll}x_{1} & x_{2} & x_{3}\end{array}\right]^{\mathrm{T}}$, the following inequality holds for any positive scalar $0<\gamma<1$.

$$
\sum_{i=1}^{3}\left|x_{i}\right|^{1+\gamma} \geq\left(\sum_{i=1}^{3}\left|x_{i}\right|^{2}\right)^{(1+\gamma) / 2} .
$$

3.1. Sliding Surface Design. Using the measurable attitude $\boldsymbol{\sigma}$ and angular velocity $\boldsymbol{\omega}$, a nonlinear sliding surface is designed as

$$
\mathbf{S}=\left[\begin{array}{lll}
s_{1} & s_{2} & s_{3}
\end{array}\right]^{\mathrm{T}}=\boldsymbol{\omega}_{e}+\lambda \mathbf{G}^{-1}\left(\boldsymbol{\sigma}_{e}\right)\left|\boldsymbol{\sigma}_{e}\right|^{\gamma} \operatorname{sgn}\left(\boldsymbol{\sigma}_{e}\right),
$$

where $\lambda$ and $0<\gamma<1$ are two positive scalars. Now we are ready to summarize the first result of this study by using the following theorem.

Theorem 4. If an appropriate control effort $\mathbf{u}$ is developed to guarantee that all the states of the attitude tracking error system (8)-(9) reach the sliding surface $\mathbf{S}$ at $t=t^{*}$ and stay on it thereafter; then it leads to $\boldsymbol{\sigma}_{e}(t) \equiv \mathbf{0}$ and $\boldsymbol{\omega}_{e}(t) \equiv \mathbf{0}$ for all $t \geq t^{*}+V_{1}^{(1-\gamma) / 2}\left(t^{*}\right) / \lambda(1-\gamma) 2^{(1-\gamma) / 2}$.

Proof. According to the sliding mode control theory, it is known that if a controller can be designed to govern the system states reaching on the sliding surface and staying on it thereafter, then it leaves (16) as

$$
\mathbf{S}(t)=\boldsymbol{\omega}_{e}+\lambda \mathbf{G}^{-1}\left(\boldsymbol{\sigma}_{e}\right)\left|\boldsymbol{\sigma}_{e}\right|^{\gamma} \operatorname{sgn}\left(\boldsymbol{\sigma}_{e}\right)=\mathbf{0}, \quad \forall t \geq t^{*} .
$$




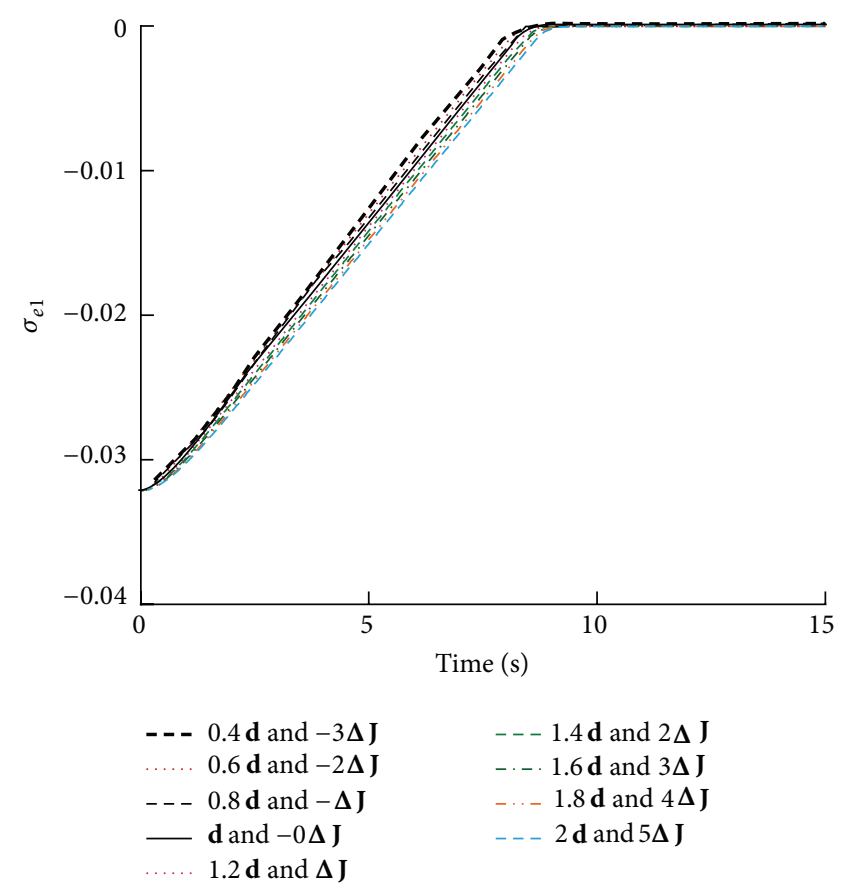

(a) The whole response

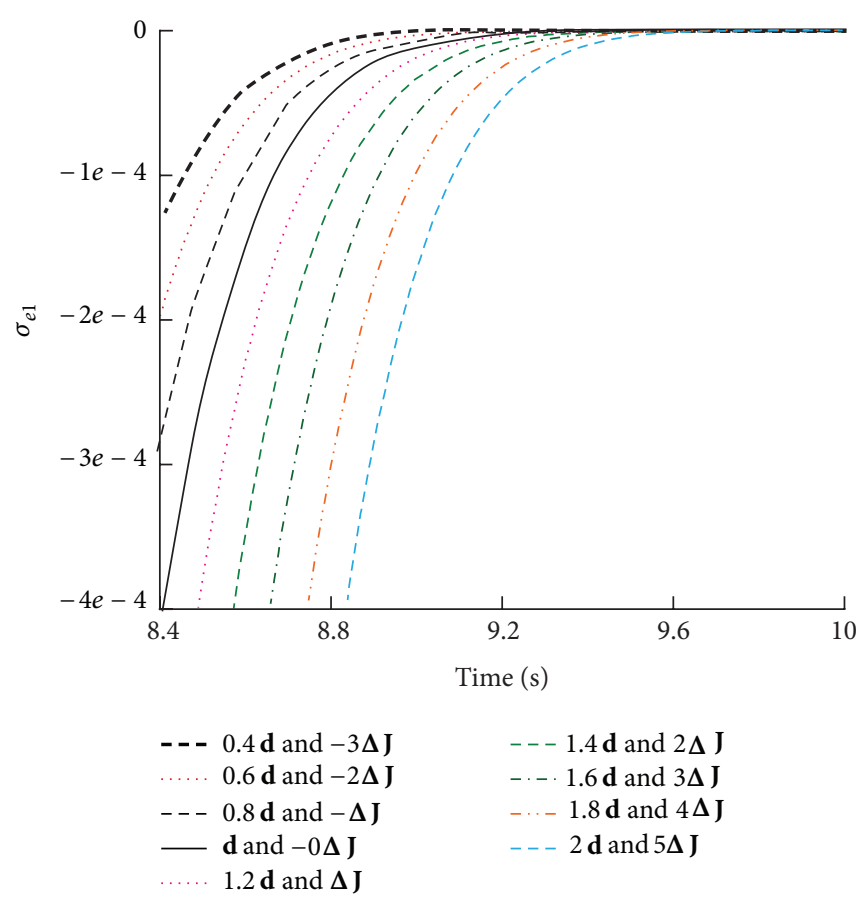

(b) The steady-state behavior

FIGURE 5: The attitude tracking error $\sigma_{e 1}$ with different disturbance and uncertain inertia.

At this time, consider the following Lyapunov candidate function:

$$
V_{1}=\frac{1}{2} \boldsymbol{\sigma}_{e}^{\mathrm{T}} \boldsymbol{\sigma}_{e}
$$

Applying (8) and (17), it can calculate the time-derivative of $V_{1}$ as

$$
\dot{V}_{1}=\boldsymbol{\sigma}_{e}^{\mathrm{T}} \dot{\boldsymbol{\sigma}}_{e}=\boldsymbol{\sigma}_{e}^{\mathrm{T}} \mathbf{G}\left(\boldsymbol{\sigma}_{e}\right) \boldsymbol{\omega}_{e}=-\lambda \boldsymbol{\sigma}_{e}^{\mathrm{T}}\left|\boldsymbol{\sigma}_{e}\right|^{\gamma} \operatorname{sgn}\left(\boldsymbol{\sigma}_{e}\right), \quad \forall t \geq t^{*} .
$$

Using (18) and (15) in Lemma 3, it yields

$$
\begin{aligned}
\dot{V}_{1} & =-\lambda \boldsymbol{\sigma}_{e}^{\mathrm{T}}\left|\boldsymbol{\sigma}_{e}\right|^{\gamma} \operatorname{sgn}\left(\boldsymbol{\sigma}_{e}\right) \leq-\lambda\left(\left\|\boldsymbol{\sigma}_{e}\right\|\right)^{1+\gamma} \\
& =-2^{(1+\gamma) / 2} \lambda V_{1}^{(1+\gamma) / 2}, \quad \forall t \geq t^{*} .
\end{aligned}
$$

Due to $0<\gamma<1$, it yields $0<(1+\gamma) / 2<1$. From Lemma 2 , it is thus obtained from inequality $(20)$ that

$$
V_{1}(t) \equiv 0, \quad t \geq t_{1}
$$

where $t_{1}$ is given by

$$
t_{1}=t^{*}+\frac{V_{1}^{(1-\gamma) / 2}\left(t^{*}\right)}{\lambda(1-\gamma) 2^{(1-\gamma) / 2}} .
$$

Consequently, it yields from (21) that $\boldsymbol{\sigma}_{e}(t) \equiv \mathbf{0}$ for all $t \geq t_{1}$. Then, it is further obtained from (17) that $\boldsymbol{\omega}_{e}(t) \equiv \mathbf{0}$ for all $t \geq t_{1}$. To this end, it completes the proof.
3.2. Finite-Time Controller Design. Due to uncertain inertia, it can be obtained from the defined sliding surface (17) and the attitude tracking error dynamics (9) that

$$
\begin{aligned}
\mathbf{J} \dot{\mathbf{S}}= & \mathbf{J}\left[\dot{\boldsymbol{\omega}}_{e}+\lambda \frac{d \mathbf{G}^{-1}\left(\boldsymbol{\sigma}_{e}\right)}{d t}\left|\boldsymbol{\sigma}_{e}\right|^{\gamma} \mathbf{s g n}\left(\boldsymbol{\sigma}_{e}\right)\right. \\
& \left.+\lambda \gamma \mathbf{G}^{-1}\left(\boldsymbol{\sigma}_{e}\right)\left|\boldsymbol{\sigma}_{e}\right|^{1-\gamma} \mathbf{G}\left(\boldsymbol{\sigma}_{e}\right) \boldsymbol{\omega}_{e}\right] \\
= & \lambda \mathbf{J} \frac{d \mathbf{G}^{-1}\left(\boldsymbol{\sigma}_{e}\right)}{d t}\left|\boldsymbol{\sigma}_{e}\right|^{\gamma} \operatorname{sgn}\left(\boldsymbol{\sigma}_{e}\right) \\
& +\lambda \gamma \mathbf{J} \mathbf{G}^{-1}\left(\boldsymbol{\sigma}_{e}\right)\left|\boldsymbol{\sigma}_{e}\right|^{\gamma-1} \mathbf{G}\left(\boldsymbol{\sigma}_{e}\right) \boldsymbol{\omega}_{e} \\
& -\left[\boldsymbol{\omega}_{e}+\mathbf{R}\left(\boldsymbol{\sigma}_{e}\right) \boldsymbol{\omega}_{d}\right]^{\times} \mathbf{J}\left(\boldsymbol{\omega}_{e}+\mathbf{R}\left(\boldsymbol{\sigma}_{e}\right) \boldsymbol{\omega}_{d}\right) \\
& +\mathbf{J}\left[\boldsymbol{\omega}_{e}^{\times} \mathbf{R}\left(\boldsymbol{\sigma}_{e}\right) \boldsymbol{\omega}_{d}-\mathbf{R}\left(\boldsymbol{\sigma}_{e}\right) \dot{\boldsymbol{\omega}}_{d}\right]+\mathbf{u}+\mathbf{d} \\
= & \mathbf{H}_{1}\left(\boldsymbol{\omega}, \boldsymbol{\omega}_{e}, \boldsymbol{\sigma}_{e}, \boldsymbol{\omega}_{d}, \mathbf{J}_{0}\right)+\mathbf{H}_{2}\left(\boldsymbol{\omega}, \boldsymbol{\omega}_{e}, \boldsymbol{\sigma}_{e}, \boldsymbol{\omega}_{d}, \mathbf{d}, \Delta \mathbf{J}\right)+\mathbf{u}
\end{aligned}
$$

where

$$
\begin{aligned}
\mathbf{H}_{1}\left(\boldsymbol{\omega}, \boldsymbol{\omega}_{e}, \boldsymbol{\sigma}_{e}, \boldsymbol{\omega}_{d}, \mathbf{J}_{0}\right) \\
=-\boldsymbol{\omega}^{\times} \mathbf{J}_{0} \boldsymbol{\omega}+\mathbf{J}_{0}\left[\boldsymbol{\omega}_{e}^{\times} \mathbf{R}\left(\boldsymbol{\sigma}_{e}\right) \boldsymbol{\omega}_{d}-\mathbf{R}\left(\boldsymbol{\sigma}_{e}\right) \dot{\boldsymbol{\omega}}_{d}\right] \\
\quad+\lambda \mathbf{J}_{0} \frac{d \mathbf{G}^{-1}\left(\boldsymbol{\sigma}_{e}\right)}{d t}\left|\boldsymbol{\sigma}_{e}\right|^{\gamma} \operatorname{sgn}\left(\boldsymbol{\sigma}_{e}\right) \\
\quad+\lambda \gamma \mathbf{J}_{0} \mathbf{G}^{-1}\left(\boldsymbol{\sigma}_{e}\right)\left|\boldsymbol{\sigma}_{e}\right|^{\gamma-1} \mathbf{G}\left(\boldsymbol{\sigma}_{e}\right) \boldsymbol{\omega}_{e}
\end{aligned}
$$




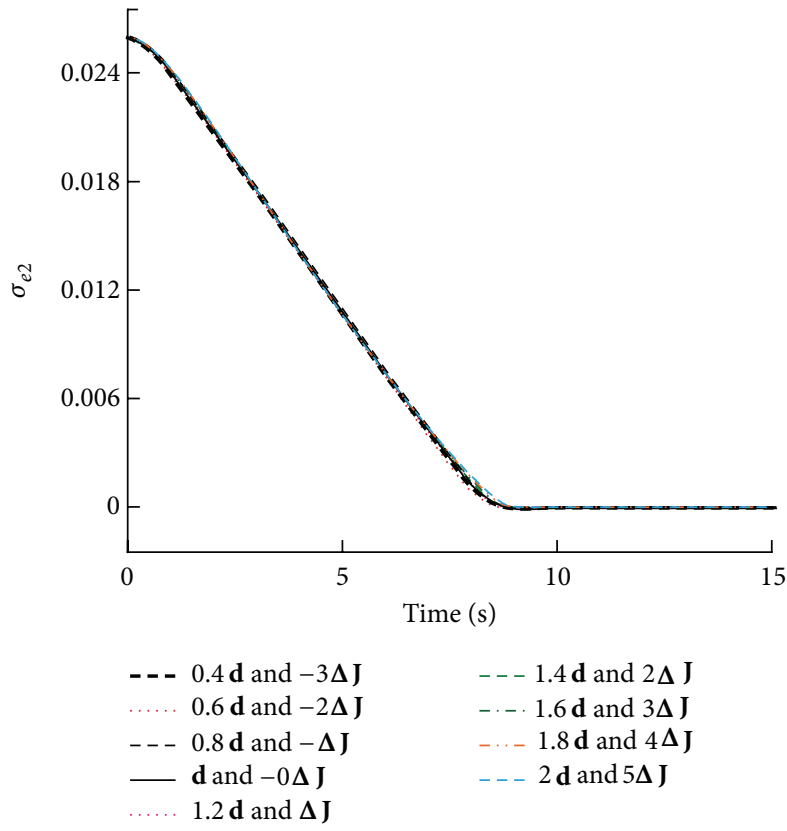

(a) The whole response

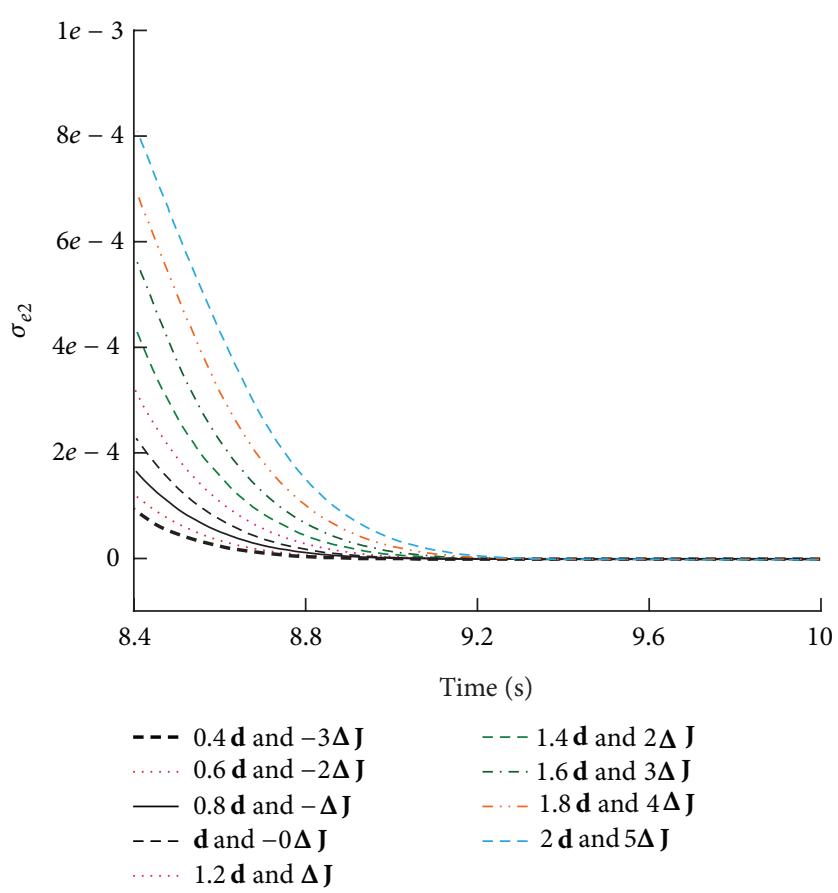

(b) The steady-state behavior

FIGURE 6: The attitude tracking error $\sigma_{e 2}$ with different disturbance and uncertain inertia.

$$
\begin{aligned}
\mathbf{H}_{2}\left(\boldsymbol{\omega}, \boldsymbol{\omega}_{e}, \boldsymbol{\sigma}_{e}, \boldsymbol{\omega}_{d}, \mathbf{d}, \Delta \mathbf{J}\right) \\
=\lambda \Delta \mathbf{J} \frac{d \mathbf{G}^{-1}\left(\boldsymbol{\sigma}_{e}\right)}{d t}\left|\boldsymbol{\sigma}_{e}\right|^{\gamma} \operatorname{sgn}\left(\boldsymbol{\sigma}_{e}\right) \\
\quad+\lambda \gamma \Delta \mathbf{J G}^{-1}\left(\boldsymbol{\sigma}_{e}\right)\left|\boldsymbol{\sigma}_{e}\right|^{\gamma-1} \mathbf{G}\left(\boldsymbol{\sigma}_{e}\right) \boldsymbol{\omega}_{e} \\
-\boldsymbol{\omega}^{\times} \Delta \mathbf{J} \boldsymbol{\omega}+\Delta \mathbf{J}\left[\boldsymbol{\omega}_{e}^{\times} \mathbf{R}\left(\boldsymbol{\sigma}_{e}\right) \boldsymbol{\omega}_{d}-\mathbf{R}\left(\boldsymbol{\sigma}_{e}\right) \dot{\boldsymbol{\omega}}_{d}\right]+\mathbf{d} .
\end{aligned}
$$

Because the angular velocity and the attitude are measurable, while the nominal inertia matrix $\mathbf{J}_{0}$ is known, the term $\mathbf{H}_{1}\left(\boldsymbol{\omega}, \boldsymbol{\omega}_{e}, \boldsymbol{\sigma}_{e}, \boldsymbol{\omega}_{d}, \mathbf{J}_{0}\right)$ in (23) can be exactly obtained. However, the uncertain term $\mathbf{H}_{2}\left(\boldsymbol{\omega}, \boldsymbol{\omega}_{e}, \boldsymbol{\sigma}_{e}, \boldsymbol{\omega}_{d}, \mathbf{d}, \Delta \mathbf{J}\right)$ in (23) cannot be obtained due to $\Delta \mathbf{J}$ and the unknown external disturbance $\mathbf{d}$.

Theorem 5. Consider the attitude tracking error system described by (8)-(9) in the presence of uncertain inertia parameters $\Delta \mathbf{J}$ and external disturbance $\mathbf{d}$; design an sliding mode controller as

$$
\mathbf{u}=-\mathbf{H}_{1}\left(\omega, \omega_{e}, \sigma_{e}, \omega_{d}, \mathbf{J}_{0}\right)-K \frac{\operatorname{sgn}(\mathbf{S})}{\|\mathbf{S}\|}-\eta \operatorname{sgn}(\mathbf{S}),
$$

where $K$ and $\eta$ are positive control gains. Suppose that $\eta$ is chosen such that

$$
\eta \geq \max _{t \in\left[t_{0},+\infty\right)}\left\|\mathbf{H}_{2}\left(\boldsymbol{\omega}, \boldsymbol{\omega}_{e}, \boldsymbol{\sigma}_{e}, \boldsymbol{\omega}_{d}, \mathbf{d}, \Delta \mathbf{J}_{0}\right)+\frac{1}{2} \frac{d \Delta \mathbf{J}(t)}{d t} \mathbf{S}\right\| .
$$

Then, the closed-loop attitude tracking error system is finitetime stable. It guarantees that the actual attitude $\sigma$ follows the desired attitude $\sigma_{d}$ in finite time.
Proof. Consider a Lyapunov candidate function for $\mathbf{S}$ as

$$
V_{2}=\frac{1}{2} \mathbf{S}^{\mathrm{T}} \mathbf{J S}
$$

Then, differentiating (27) and inserting (23) and the controller Equation (25) yield

$$
\begin{aligned}
\dot{V}_{2}= & \mathbf{S}^{\mathrm{T}} \mathbf{J} \dot{\mathbf{S}}+\frac{1}{2} \mathbf{S}^{\mathrm{T}} \frac{d \Delta \mathbf{J}(t)}{d t} \mathbf{S} \\
=\mathbf{S}^{\mathrm{T}} & {\left[\mathbf{H}_{1}\left(\boldsymbol{\omega}, \boldsymbol{\omega}_{e}, \boldsymbol{\sigma}_{e}, \boldsymbol{\omega}_{d}, \mathbf{J}_{0}\right)+\mathbf{H}_{2}\left(\boldsymbol{\omega}, \boldsymbol{\omega}_{e}, \boldsymbol{\sigma}_{e}, \boldsymbol{\omega}_{d}, \mathbf{d}, \Delta \mathbf{J}\right)\right.} \\
& \left.\quad+\mathbf{u}+\frac{1}{2} \frac{d \Delta \mathbf{J}(t)}{d t} \mathbf{S}\right] \\
= & \mathbf{S}^{\mathrm{T}}\left[\mathbf{H}_{2}\left(\boldsymbol{\omega}, \boldsymbol{\omega}_{e}, \boldsymbol{\sigma}_{e}, \boldsymbol{\omega}_{d}, \mathbf{d}, \Delta \mathbf{J}\right)-K \frac{\mathbf{s g n}(\mathbf{S})}{\|\mathbf{S}\|}\right. \\
\leq & \left.-\eta \mathbf{s g n}(\mathbf{S})+\frac{1}{2} \frac{d \Delta \mathbf{J}(t)}{d t} \mathbf{S}\right] \\
& \times\left\|\mathbf{H}_{2}\left(\boldsymbol{\omega}, \boldsymbol{\omega}_{e}, \boldsymbol{\sigma}_{e}, \boldsymbol{\omega}_{d}, \mathbf{d}, \Delta \mathbf{J}\right)+\frac{1}{2} \frac{d \Delta \mathbf{J}(t)}{d t} \mathbf{S}\right\|-\eta\|\mathbf{S}\| .
\end{aligned}
$$

With the choice of the control gain in (26), it leaves (28) as

$$
\dot{V}_{2} \leq-K
$$

Integrating (29) from $t_{0}$ to $t$ yields

$$
\int_{t_{0}}^{t} \dot{V}_{2} d s=V_{2}(t)-V_{2}\left(t_{0}\right) \leq-\int_{t_{0}}^{t} K d t=-K\left(t-t_{0}\right) .
$$




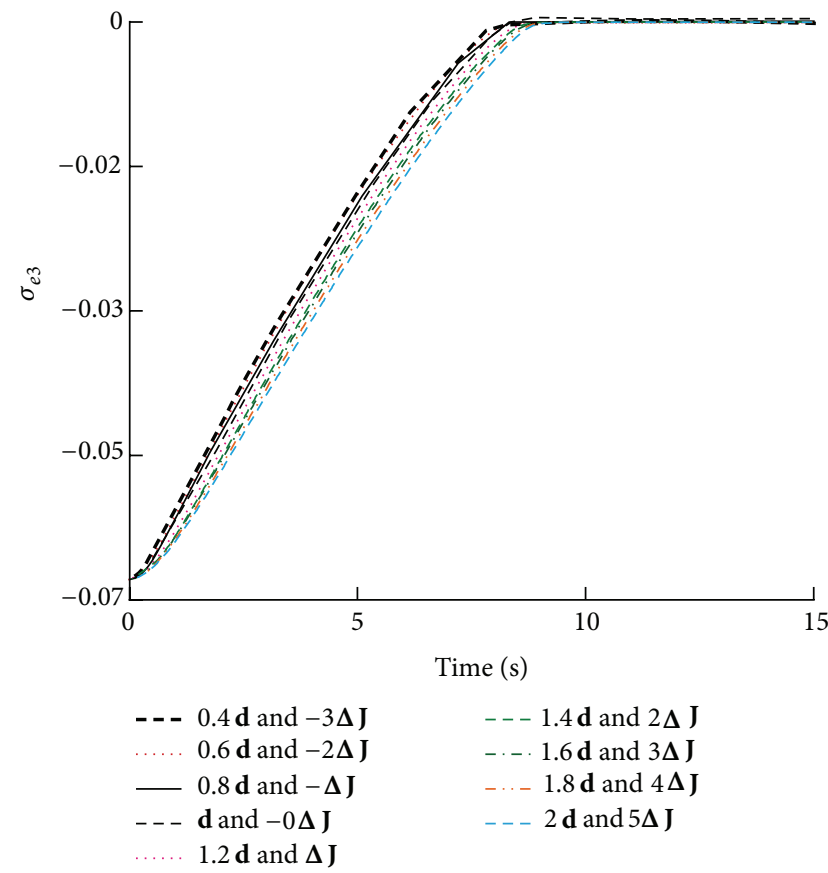

(a) The whole response

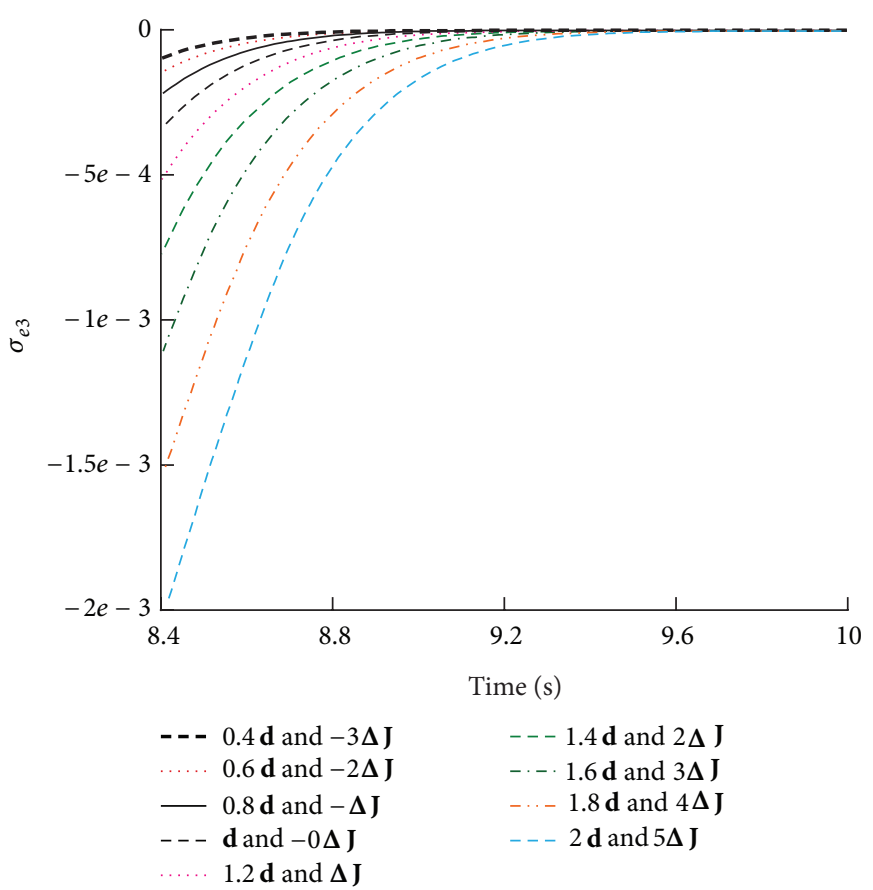

(b) The steady-state behavior

FIGURE 7: The attitude tracking error $\sigma_{e 3}$ with different disturbance and uncertain inertia.

Solving the inequality (30), it follows from the positive definiteness of $V_{2}$ that

$$
V_{2}(t) \equiv 0, \quad \forall t \geq t^{*}
$$

where

$$
t^{*}=t_{0}+\frac{V_{2}\left(t_{0}\right)}{K}
$$

Consequently, it can be concluded from (31) that all the states of the attitude tracking error system will reach the sliding surface $\mathbf{S}$ at $t=t^{*}$. Moreover, starting at the time $t=t^{*}$, the states will stay on the sliding surface and thereafter. At this time, $\boldsymbol{\sigma}_{e}(t) \equiv \mathbf{0}$ and $\boldsymbol{\omega}_{e}(t) \equiv \mathbf{0}$ can be obtained from Theorem 4 for all the time $t \geq T_{1} \triangleq t_{0}+$ $V_{2}\left(t_{0}\right) / K+V_{1}^{(1-\gamma) / 2}\left(t^{*}\right) / \lambda(1-\gamma) 2^{(1-\gamma) / 2}$. Hence, it can be further concluded from Definition 1 that the attitude tracking error system is finite-time stable. Moreover, the time needed to accomplish the attitude tracking maneuver is $\Delta t$, which is given by

$$
\Delta t=\frac{V_{2}\left(t_{0}\right)}{K}+\frac{V_{1}^{(1-\gamma) / 2}\left(t^{*}\right)}{\lambda(1-\gamma) 2^{(1-\gamma) / 2}} .
$$

3.3. Adaptive Sliding Mode Control with Finite-Time Convergence. It is seen in Theorem 4 that the control gain $\eta$ is actually chosen to be larger than the upper bound on the lumped uncertainty (the combined effect induced by $\mathbf{H}_{2}\left(\boldsymbol{\omega}, \boldsymbol{\omega}_{e}, \boldsymbol{\sigma}_{e}, \boldsymbol{\omega}_{d}, \mathbf{d}, \Delta \mathbf{J}_{0}\right)$ and $\left.(1 / 2)(d \Delta \mathbf{J}(t) / d t) \mathbf{S}\right)$. It may be difficult to obtain the upper bound in practical aerospace engineering. As a sequence, an adaptive sliding mode control scheme will be proposed to achieve finite-time attitude tracking in this subsection. The adaptive control technique will be applied to estimate such bound.

Although there exist uncertain inertia parameters and external disturbance acting on the satellite, those uncertainties are bound in practice. Therefore, it is reasonable to make the following assumptions.

Assumption 6. The external disturbance $\mathbf{d}$ is bounded by $\|\mathbf{d}\| \leq d_{0}$, where $d_{0}$ is a positive but unknown scalar.

Assumption 7. There exists a constant (unknown) $d_{1} \geq 0$ such that $\|\Delta \mathbf{J}\| \leq d_{1}$.

Assumption 8. The changing rate of $\Delta \mathrm{J}$ is bounded, that is, $\|d \Delta \mathbf{J}(t) / d t\| \leq d_{2}$ for some unknown constant $d_{2} \geq 0$.

For any on-orbit satellite, gravity-gradient torque, aerodynamic torque, solar radiation torque, and earth magnetic torque are the primary external disturbance for $\mathbf{d}$ [44]. Those disturbances are bounded in practice. Therefore, Assumption 6 is reasonable. On the other hand, if the changing rate of $\Delta \mathbf{J}$ is infinite, that is, $\|d \Delta \mathbf{J}(t) / d t\|=+\infty$, then it may lead to $\|\Delta \mathbf{J}\|=+\infty$ after certain time. That is impossible for practical satellite. It is thus practical to make Assumption 8.

Using $\left\|\mathbf{R}\left(\boldsymbol{\sigma}_{e}\right)\right\|=1$, the following inequalities can be obtained from (7) and Assumption 7:

$$
\begin{gathered}
\left\|\boldsymbol{\omega}^{\times} \Delta \mathbf{J} \boldsymbol{\omega}\right\| \leq\left\|\boldsymbol{\omega}^{\times}\right\|\|\Delta \mathbf{J}\|\|\boldsymbol{\omega}\| \leq d_{1}\|\boldsymbol{\omega}\|^{2}, \\
\left\|\Delta \mathbf{J}\left[\boldsymbol{\omega}_{e}^{\times} \mathbf{R}\left(\boldsymbol{\sigma}_{e}\right) \boldsymbol{\omega}_{d}-\mathbf{R}\left(\boldsymbol{\sigma}_{e}\right) \dot{\boldsymbol{\omega}}_{d}\right]\right\|
\end{gathered}
$$




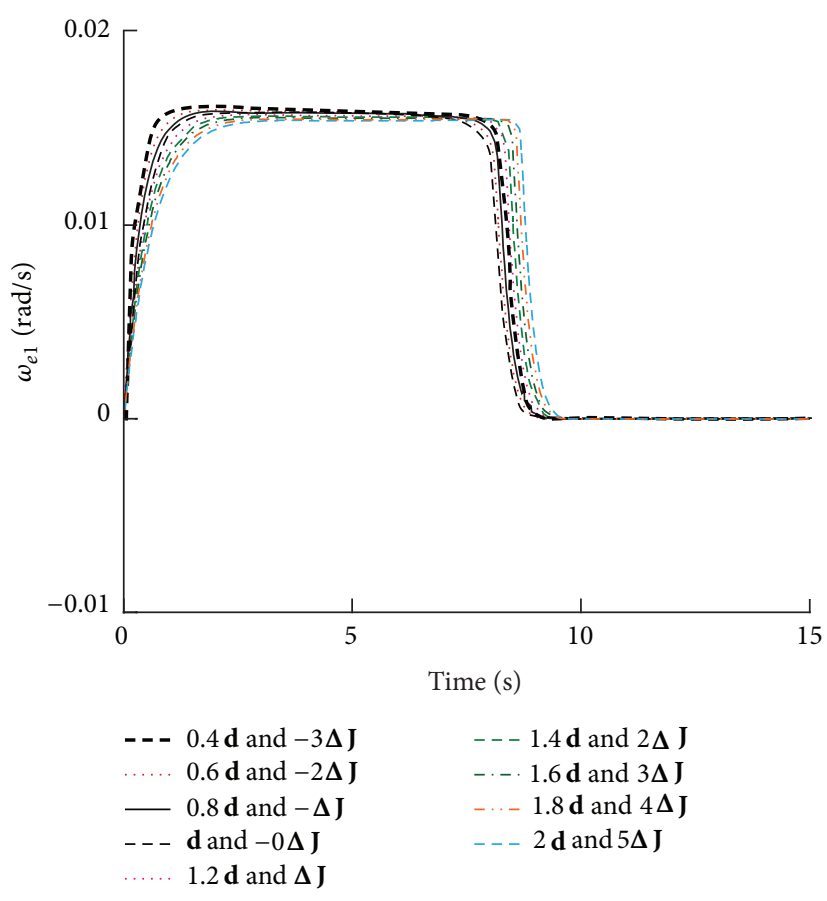

(a) The whole response

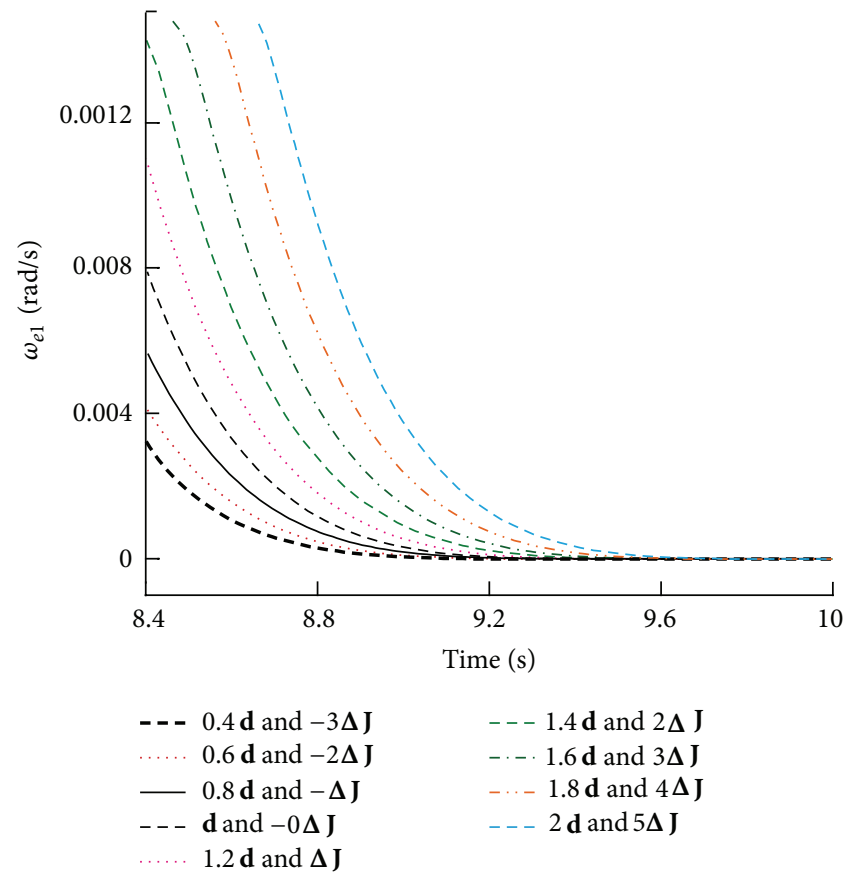

(b) The steady-state behavior

FIGURE 8: The angular velocity tracking error $\omega_{e 1}$ with different disturbance and uncertain inertia.

$$
\begin{gathered}
\leq d_{1}\left[\left\|\boldsymbol{\omega}-\mathbf{R}\left(\boldsymbol{\sigma}_{e}\right) \boldsymbol{\omega}_{d}\right\|\left\|\mathbf{R}\left(\boldsymbol{\sigma}_{e}\right)\right\|\left\|\boldsymbol{\omega}_{d}\right\|\right. \\
\left.+\left\|\mathbf{R}\left(\boldsymbol{\sigma}_{e}\right)\right\|\left\|\dot{\boldsymbol{\omega}}_{d}\right\|\right] \\
\leq d_{1}\left[\left\|\boldsymbol{\omega}_{d}\right\|\left(\|\boldsymbol{\omega}\|+\left\|\boldsymbol{\omega}_{d}\right\|\right)+\left\|\dot{\boldsymbol{\omega}}_{d}\right\|\right] .
\end{gathered}
$$

Additionally, from (10), one has

$$
\left\|\mathrm{G}\left(\boldsymbol{\sigma}_{\mathrm{e}}\right)\right\|=\frac{1+\boldsymbol{\sigma}_{e}^{\mathrm{T}} \boldsymbol{\sigma}_{e}}{4}, \quad\left\|\mathrm{G}^{-1}\left(\boldsymbol{\sigma}_{e}\right)\right\|=\frac{4}{1+\boldsymbol{\sigma}_{e}^{\mathrm{T}} \boldsymbol{\sigma}_{e}} \leq 4
$$

Then,

$$
\begin{gathered}
\left\|\Delta \mathbf{J} \frac{d \mathbf{G}^{-1}\left(\boldsymbol{\sigma}_{e}\right)}{d t}\left|\boldsymbol{\sigma}_{e}\right|^{\gamma} \operatorname{sgn}\left(\boldsymbol{\sigma}_{e}\right)\right\| \\
\leq d_{1}\left\|\frac{d \mathbf{G}^{-1}\left(\boldsymbol{\sigma}_{e}\right)}{d t}\right\|\left\|\left|\boldsymbol{\sigma}_{\mathbf{e}}\right|^{\gamma} \operatorname{sgn}\left(\boldsymbol{\sigma}_{e}\right)\right\|, \\
\left\|\Delta \mathbf{J G}^{-1}\left(\boldsymbol{\sigma}_{e}\right)\left|\boldsymbol{\sigma}_{e}\right|^{\gamma-1} \mathbf{G}\left(\boldsymbol{\sigma}_{e}\right) \boldsymbol{\omega}_{e}\right\| \\
\leq d_{1}\left\|\left|\boldsymbol{\sigma}_{e}\right|^{\gamma-1}\right\|\left(\|\boldsymbol{\omega}\|+\left\|\boldsymbol{\omega}_{d}\right\|\right) .
\end{gathered}
$$

Also, using Assumption 8, (35), and (16) results in

$$
\begin{aligned}
\left\|\frac{d \Delta \mathbf{J}(t)}{d t} \mathbf{S}\right\| & \leq d_{2}\left\|\boldsymbol{\omega}_{e}+\lambda \mathbf{G}^{-1}\left(\boldsymbol{\sigma}_{e}\right)\left|\boldsymbol{\sigma}_{e}\right|^{\gamma} \operatorname{sgn}\left(\boldsymbol{\sigma}_{e}\right)\right\| \\
& \leq d_{2}\left(\|\boldsymbol{\omega}\|+\left\|\boldsymbol{\omega}_{d}\right\|+4 \lambda\left\|\left.\boldsymbol{\sigma}_{e}\right|^{\gamma} \operatorname{sgn}\left(\boldsymbol{\sigma}_{e}\right)\right\|\right) .
\end{aligned}
$$

To this end, it can be found from inequalities (34), (36)-(38), and Assumption 6 that $\mathbf{H}_{2}\left(\boldsymbol{\omega}, \boldsymbol{\omega}_{e}, \boldsymbol{\sigma}_{e}, \boldsymbol{\omega}_{d}, \mathbf{d}, \Delta \mathbf{J}_{0}\right)+$ $(1 / 2)(d \Delta \mathbf{J}(t) / d t) \mathbf{S}$ is bounded by

$$
\begin{aligned}
& \left\|\mathbf{H}_{2}\left(\boldsymbol{\omega}, \boldsymbol{\omega}_{e}, \boldsymbol{\sigma}_{e}, \boldsymbol{\omega}_{d}, \mathbf{d}, \Delta \mathbf{J}_{0}\right)+\frac{1}{2} \frac{d \Delta \mathbf{J}(t)}{d t} \mathbf{S}\right\| \\
& \leq\left\|\mathbf{H}_{2}\left(\boldsymbol{\omega}, \boldsymbol{\omega}_{e}, \boldsymbol{\sigma}_{e}, \boldsymbol{\omega}_{d}, \mathbf{d}, \Delta \mathbf{J}_{0}\right)\right\|+\frac{1}{2}\left\|\frac{d \Delta \mathbf{J}(t)}{d t} \mathbf{S}\right\| \\
& \leq d_{0}+\frac{d_{2}}{2}\left(\|\boldsymbol{\omega}\|+\left\|\boldsymbol{\omega}_{d}\right\|+4 \lambda\left\|\left.\boldsymbol{\sigma}_{e}\right|^{\gamma} \operatorname{sgn}\left(\boldsymbol{\sigma}_{e}\right)\right\|\right) \\
& +d_{1} \underbrace{\left\{\begin{array}{c}
\lambda\left\|\frac{d \mathbf{G}^{-1}\left(\boldsymbol{\sigma}_{e}\right)}{d t}\right\|\left\|\left|\boldsymbol{\sigma}_{e}\right|^{\gamma} \operatorname{sgn}\left(\boldsymbol{\sigma}_{e}\right)\right\| \\
+\lambda \gamma\left\|\left|\boldsymbol{\sigma}_{e}\right|^{\gamma-1}\right\|\left(\|\boldsymbol{\omega}\|+\left\|\boldsymbol{\omega}_{d}\right\|\right)+\left\|\dot{\boldsymbol{\omega}}_{d}\right\| \\
+\|\boldsymbol{\omega}\|^{2}+\left\|\boldsymbol{\omega}_{d}\right\|\|\boldsymbol{\omega}\|+\|\|^{2}
\end{array}\right\}}_{\mathbf{H}_{3}\left(\boldsymbol{\omega}, \boldsymbol{\sigma}_{e}, \boldsymbol{\omega}_{d}\right)}
\end{aligned}
$$

$$
=\psi^{\mathrm{T}} \mathrm{D}
$$

where $\mathbf{D}=\left[\begin{array}{lll}d_{0} & d_{1} & d_{2}\end{array}\right]^{\mathrm{T}} \in \mathfrak{R}^{3}, \psi=$ $\left[\begin{array}{lll}1 & \mathbf{H}_{3}\left(\boldsymbol{\omega}, \boldsymbol{\sigma}_{e}, \boldsymbol{\omega}_{d}\right) & \left(\|\boldsymbol{\omega}\|+\left\|\boldsymbol{\omega}_{d}\right\|+4 \lambda\left\|\left.\boldsymbol{\sigma}_{e}\right|^{\gamma} \operatorname{sgn}\left(\boldsymbol{\sigma}_{e}\right)\right\|\right) / 2\end{array}\right]^{\mathrm{T}}$. Here, $\mathbf{D}$ is a constant vector but unknown, while $\psi$ is known, and each element of $\psi$ is positive. Now, we are ready to summarize the finite-time control solution to underlying attitude tracking problem by using adaptive control technique.

Theorem 9. For an attitude system described by (1)-(2) of a rigid satellite subject to uncertain inertia parameters and 


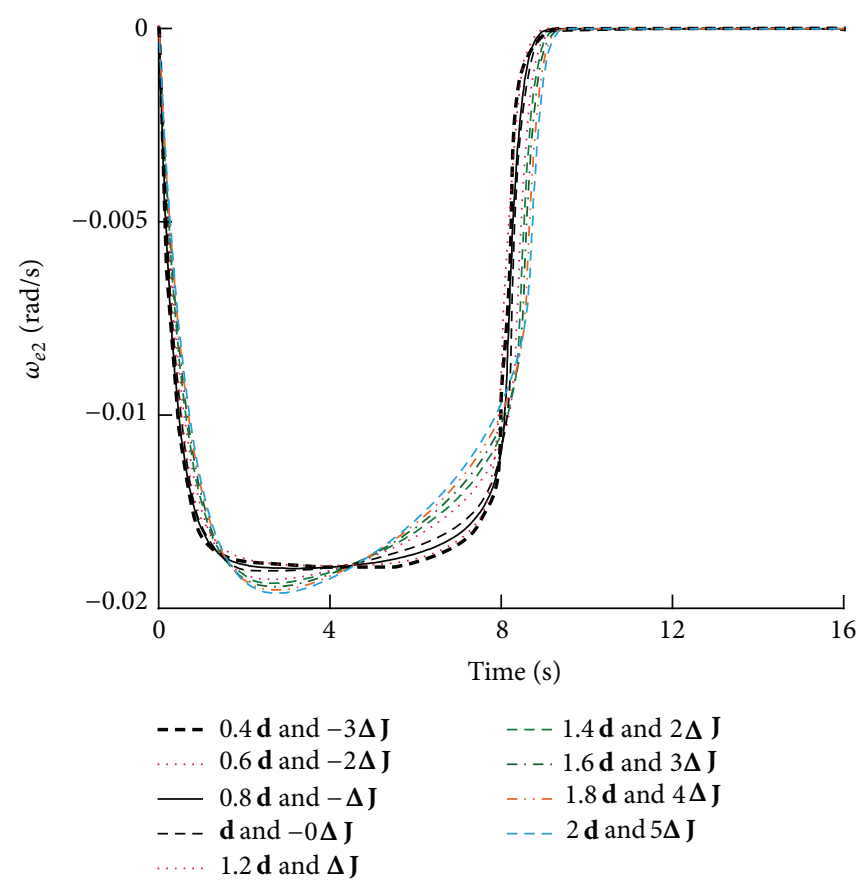

(a) The whole response

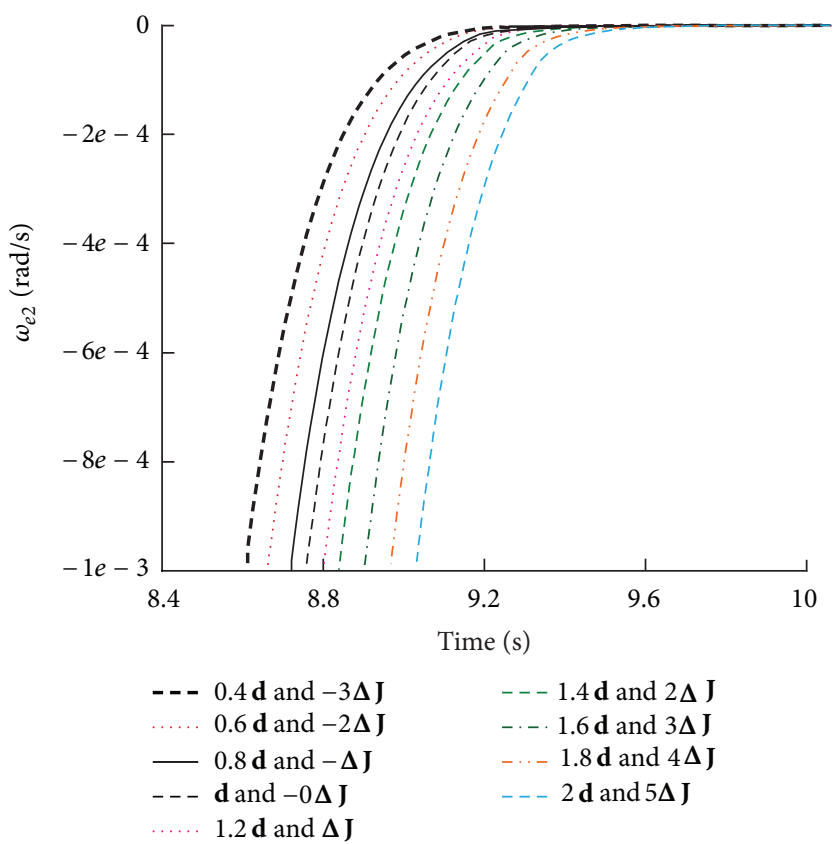

(b) The steady-state behavior

FIGURE 9: The angular velocity tracking error $\omega_{e 2}$ with different disturbance and uncertain inertia.

unknown external disturbance, if the following adaptive sliding mode controller is designed and applied

$$
\mathbf{u}=-\mathbf{H}_{1}\left(\boldsymbol{\omega}, \omega_{e}, \boldsymbol{\sigma}_{e}, \omega_{d}, \mathbf{J}_{0}\right)-K \frac{\mathbf{s g n}(\mathbf{S})}{\|\mathbf{S}\|}-\psi^{\mathrm{T}} \widehat{\operatorname{D}} \operatorname{sgn}(\mathbf{S}),
$$

where $\widehat{\mathbf{D}} \in \mathfrak{R}^{3}$ is the estimate of $\mathbf{D}$, and it is updated by the adaptive law

$$
\dot{\widehat{\mathbf{D}}}=\pi \psi^{\mathrm{T}}\|\mathbf{S}\|
$$

with $\pi$ is positive scalar, then, the attitude tracking maneuver is accomplished in finite time; the closed-loop attitude tracking error system is finite-time stable. Moreover, the control objective as stated in Section 2.3 is achieved.

Proof. The proof uses elements of Lyapunov stability theory and is organized as follows.

(A) Finite-Time Stability of the Closed-Loop Attitude Tracking Error System. Consider a candidate Lyapunov function of the form:

$$
V_{3}=\frac{1}{2} \mathbf{S}^{\mathrm{T}} \mathbf{J S}+\frac{\widetilde{\mathbf{D}}^{\mathrm{T}} \widetilde{\mathbf{D}}}{2 \pi}
$$

where $\widetilde{\mathbf{D}}=\mathbf{D}-\widehat{\mathbf{D}}$ denotes the estimate error between $\mathbf{D}$ and $\widehat{D}$.
Using (39), it thus follows that

$$
\begin{aligned}
\dot{V}_{3}= & \mathbf{S}^{\mathrm{T}} \mathbf{J} \dot{\mathbf{S}}+\frac{1}{2} \mathbf{S}^{\mathrm{T}} \frac{d \Delta \mathbf{J}(t)}{d t} \mathbf{S}-\frac{\widetilde{\mathbf{D}}^{\mathrm{T}} \dot{\hat{\mathbf{D}}}}{\pi} \\
= & \mathbf{S}^{\mathrm{T}}\left[\mathbf{H}_{1}\left(\boldsymbol{\omega}, \boldsymbol{\omega}_{e}, \boldsymbol{\sigma}_{e}, \boldsymbol{\omega}_{d}, \mathbf{J}_{0}\right)+\mathbf{H}_{2}\left(\boldsymbol{\omega}, \boldsymbol{\omega}_{e}, \boldsymbol{\sigma}_{e}, \boldsymbol{\omega}_{d}, \mathbf{d}, \Delta \mathbf{J}\right)\right. \\
& \left.+\mathbf{u}+\frac{1}{2} \frac{d \Delta \mathbf{J}(t)}{d t} \mathbf{S}\right]-\frac{\widetilde{\mathbf{D}}^{\mathrm{T}} \dot{\widehat{\mathbf{D}}}}{\pi} \\
\leq & \|\mathbf{S}\|\left\|\mathbf{H}_{2}\left(\boldsymbol{\omega}, \boldsymbol{\omega}_{e}, \boldsymbol{\sigma}_{e}, \boldsymbol{\omega}_{d}, \mathbf{J}_{0}\right)+\frac{1}{2} \frac{d \Delta \mathbf{J}(t)}{d t} \mathbf{S}\right\| \\
& +\mathbf{S}^{\mathrm{T}}\left[\mathbf{H}_{1}\left(\boldsymbol{\omega}, \boldsymbol{\omega}_{e}, \boldsymbol{\sigma}_{e}, \boldsymbol{\omega}_{d}, \mathbf{J}_{0}\right)+\mathbf{u}\right]-\frac{\widetilde{\mathbf{D}}^{\mathrm{T}} \dot{\hat{\mathbf{D}}}}{\pi} \\
= & \boldsymbol{\psi}^{\mathrm{T}} \mathbf{D}\|\mathbf{S}\|+\mathbf{S}^{\mathrm{T}}\left[\mathbf{H}_{1}\left(\boldsymbol{\omega}, \boldsymbol{\omega}_{e}, \boldsymbol{\sigma}_{e}, \boldsymbol{\omega}_{d}, \mathbf{J}_{0}\right)+\mathbf{u}\right]-\frac{\widetilde{\mathbf{D}}^{\mathrm{T}} \dot{\hat{\mathbf{D}}}}{\pi} .
\end{aligned}
$$

Substituting the control law Equation (40) and the update law Equation (41) into (43), it yields

$$
\begin{aligned}
\dot{V}_{3} & \leq \psi^{\mathrm{T}} \mathbf{D}\|\mathbf{S}\|+\mathbf{S}^{\mathrm{T}}\left[-K \frac{\mathbf{s g n}(\mathbf{S})}{\|\mathbf{S}\|}-\psi^{\mathrm{T}} \widehat{\mathbf{D}} \operatorname{sgn}(\mathbf{S})\right]-\frac{\widetilde{\mathbf{D}}^{\mathrm{T}} \dot{\widehat{\mathbf{D}}}}{\pi} \\
& \leq-K+\widetilde{\mathbf{D}}^{\mathrm{T}} \psi\|\mathbf{S}\|-\frac{\widetilde{\mathbf{D}}^{\mathrm{T}} \dot{\hat{\mathbf{D}}}}{\pi} \\
& =-K .
\end{aligned}
$$




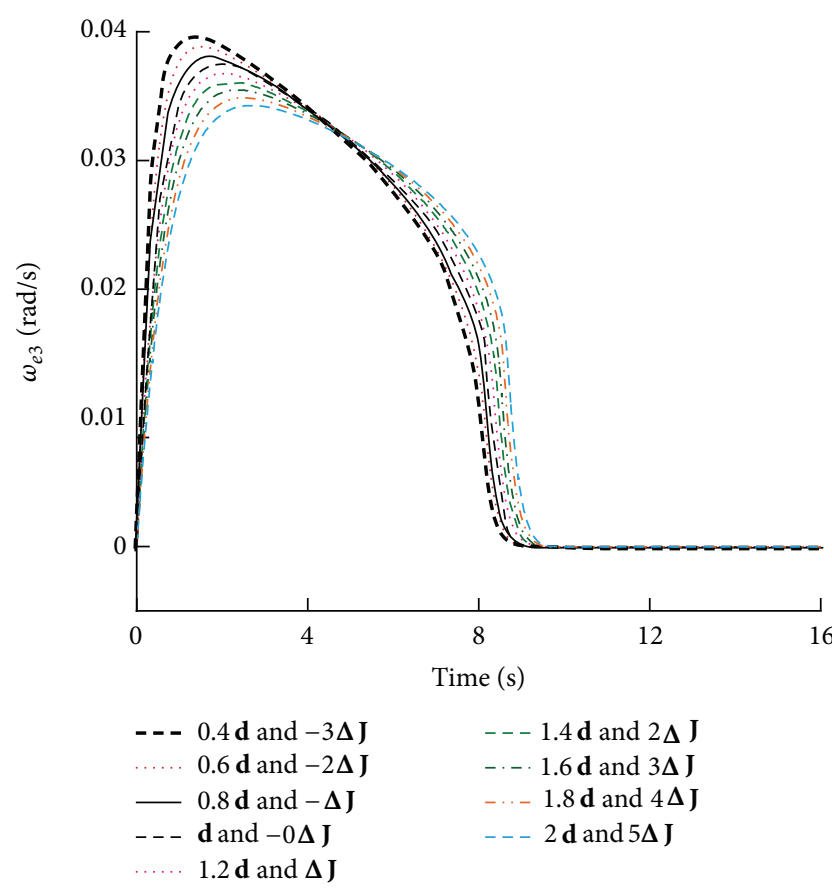

(a) The whole response

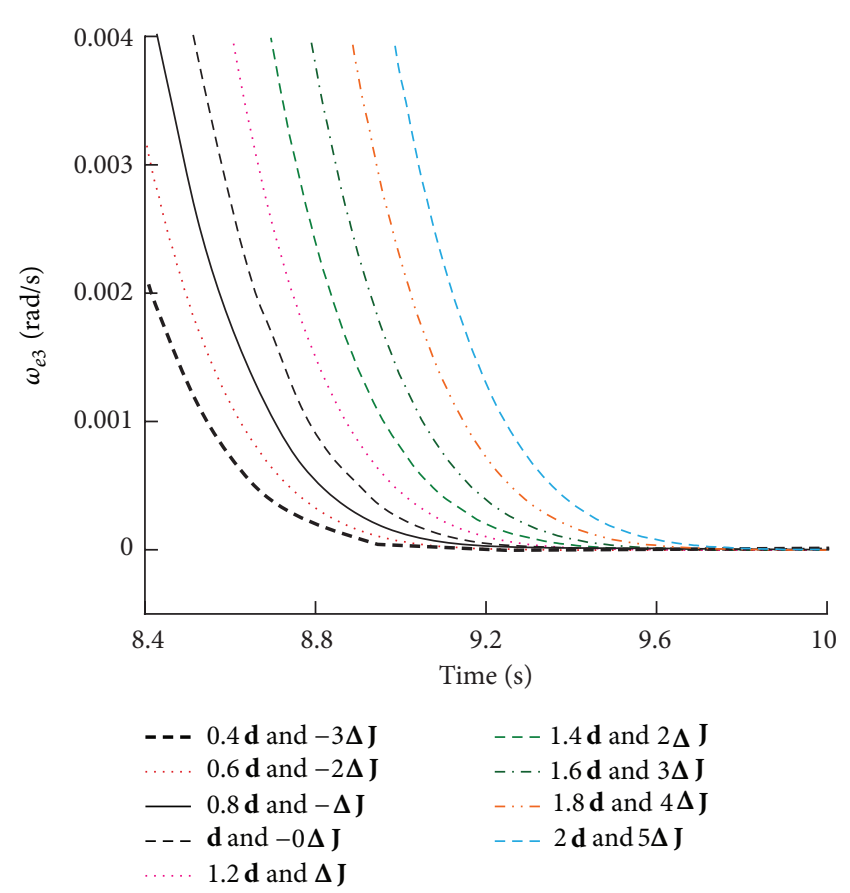

(b) The steady-state behavior

FIGURE 10: The angular velocity tracking error $\omega_{e 3}$ with different disturbance and uncertain inertia.

Integrating (44) from $t_{0}$ to $t$ yields

$$
\int_{t_{0}}^{t} \dot{V}_{3} d s=V_{3}(t)-V_{3}\left(t_{0}\right) \leq-\int_{t_{0}}^{t} K d t=-K\left(t-t_{0}\right) .
$$

Hence, it is obtained from inequality $(45)$ that $V_{3}(t) \equiv 0$ for all the $t \geq t^{*}$. Here, $t^{*}$ is given by

$$
t^{*}=t_{0}+\frac{V_{3}\left(t_{0}\right)}{K} \text {. }
$$

By using the same analysis as in the proof of Theorem 5 , it can be concluded from (45)-(46) that the attitude tracking error system is finite-time stable. The attitude tracking error $\boldsymbol{\sigma}_{e}(t)$ and the angular velocity $\boldsymbol{\omega}_{e}(t)$ are asymptotically stabilized after finite-time $t_{f}$; that is, $\boldsymbol{\sigma}_{e}(t)=\mathbf{0}, \boldsymbol{\omega}_{e}(t)=\mathbf{0}, t \geq t_{f}$,

$$
t_{f}=t_{0}+\frac{V_{3}\left(t_{0}\right)}{K}+\frac{V_{1}^{(1-\gamma) / 2}\left(t^{*}\right)}{\lambda(1-\gamma) 2^{(1-\gamma) / 2}} .
$$

Hence, the attitude tracking maneuver can be successfully accomplished in a finite-time $\Delta t$ :

$$
\Delta t=t_{f}-t_{0}=\frac{V_{3}\left(t_{0}\right)}{K}+\frac{V_{1}^{(1-\gamma) / 2}\left(t^{*}\right)}{\lambda(1-\gamma) 2^{(1-\gamma) / 2}} .
$$

(B) Finite-Time Stability of the Estimate Error $\widetilde{\mathbf{D}}$. As analyzed in (A), the controller Equation (40) guarantees that $V_{3}(t) \equiv 0$ for all the $t \geq t^{*}$. According to the definition of $V_{3}$, it can be obtained that

$$
\widetilde{\mathbf{D}}_{3}=\mathbf{0}, \quad t \geq t^{*}
$$

Hence, it can be concluded from Definition 1 that the estimate error $\widetilde{\mathbf{D}}$ is finite-time stable. Accordingly, the unknown vector $\mathbf{D}$ can be exactly estimated by $\widehat{\mathbf{D}}$ within a finite time of $\delta t=$ $t^{*}-t_{0}$.

Summarizing the analysis as presented in (A)-(B), the proof of Theorem 9 is completed.

Remark 10. It is seen in Theorem 9 that, the attitude tracking maneuver can be successfully accomplished in a finite-time $\Delta t$. It thus leads the proposed controller Equation (40) to be a good option for a satellite demanding high real-time. Moreover, it is further obtained from (48) that larger $K, \lambda, \pi$, and $(1-\gamma) 2^{(1-\gamma) / 2}$ will lead to shorter $\Delta t$. The attitude tracking can be finished in a shorter time.

\section{Numerical Example}

In this section the properties of the proposed finite-time attitude tracking control approach is evaluated through numerical simulations. A rigid satellite currently developed is numerically simulated. The orbit of the satellite is circular, with an altitude of $550 \mathrm{~km}$ and an inclination of 90.4 degrees. The satellite is with the nominal inertia parameters as $\mathbf{J}_{0}=\left[\begin{array}{ccc}20 & 2 & 0.9 \\ 2 & 17 & 0.5 \\ 0.9 & 0.5 & 15\end{array}\right] \mathrm{kg} \cdot \mathrm{m}^{2}$. A square-wave disturbance is added to each axis with periods 40, 50, and 70 seconds, and magnitudes $0.01,0.05$, and $0.08 \mathrm{Nm}$, respectively. At time $t=0$, the initial attitude of the satellite is $\sigma(0)=\left[\begin{array}{lll}-0.0321 & 0.0260 & -0.0626\end{array}\right]^{\mathrm{T}}$, the initial body angular velocity of $\boldsymbol{\omega}(0)=\left[\begin{array}{lll}0 & 0 & 0\end{array}\right]^{\mathrm{T}} \mathrm{rad} / \mathrm{sec}$. 


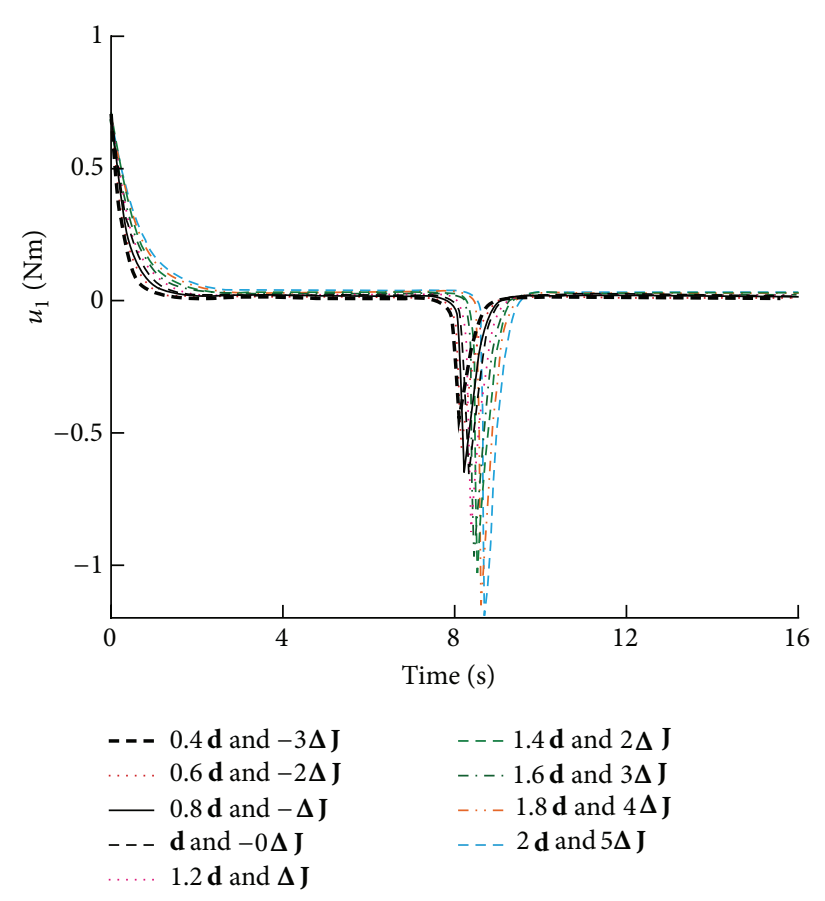

(a) The whole response

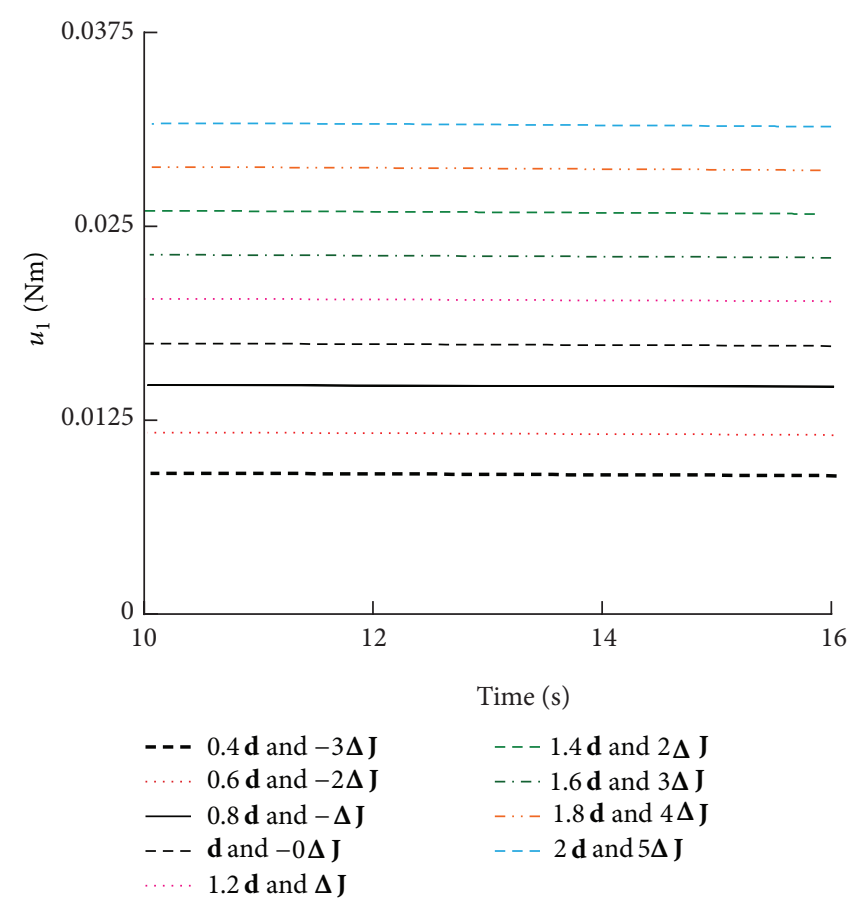

(b) The steady-state behavior

FIGURE 11: The control effort $u_{1}$ with different disturbance and uncertain inertia.

To accomplish the planned aerospace mission, an attitude tracking maneuver needs to be performed. The desired attitude motion is with time-varying desired angular velocity of the form

$$
\boldsymbol{\omega}_{d}=\left[\begin{array}{lll}
0.03 \sin \left(\frac{2 \pi t}{400}\right) & 0.03 \sin \left(\frac{2 \pi t}{600}\right) & 0.03 \sin \left(\frac{2 \pi t}{500}\right)
\end{array}\right]^{\mathrm{T}} \mathrm{rad} / \mathrm{sec}
$$

and the desired attitude trajectory $\sigma_{d}$ is obtained by using the dynamics $\dot{\boldsymbol{\sigma}}_{d}=\mathbf{G}\left(\boldsymbol{\sigma}_{d}\right) \boldsymbol{\omega}_{d}$.

When implementing the proposed adaptive sliding mode controller Equation (40), a practical problem has to be considered, namely, the chattering effect. Because it is impossible to switch the control at infinite rate, the trajectory of an SMC system chatters with respect to the sliding surface. This chattering is practically undesirable since it may excite the neglected high frequency dynamics. One practical approach to reduce the chattering is to replace the discontinuous function $\operatorname{sgn}(\mathbf{S}) /\|\mathbf{S}\|$ and $\operatorname{sgn}(\mathbf{S})$ in (40) by a continuous approximation such as $\mathbf{S} /(\|\mathbf{S}\|+\varepsilon)$ where $\varepsilon$ is a small positive constant [21]. Therefore, the control Equation (40) is modified as follows to reduce the chattering:

$$
\mathbf{u}=-\mathbf{H}_{1}\left(\boldsymbol{\omega}, \boldsymbol{\omega}_{e}, \boldsymbol{\sigma}_{e}, \boldsymbol{\omega}_{d}, \mathbf{J}_{0}\right)-K \frac{\mathbf{S}}{\|\mathbf{S}\|^{2}+\varepsilon}-\psi^{\mathrm{T}} \widehat{\mathbf{D}} \frac{\mathbf{S}}{\|\mathbf{S}\|} .
$$

Moreover, the control gains for the controller Equation (51) are chosen as $\lambda=1.5, \gamma=0.85, K=1.25$, and $\pi=0.15$.

Assume that uncertain inertia $\Delta \mathrm{J}$ is equal to $10 \%$ of the nominal value; that is, $\Delta \mathbf{J}=10 \% \mathbf{J}_{0}$. To verify the performance of the developed approach, simulation is carried out for the satellite subject to different disturbances and uncertain inertia matrix. The case "Nd and $c \Delta \mathbf{J}$ " means that the external disturbance acting on the satellite is $N \mathbf{d}$, while the uncertain inertia is $c \Delta \mathbf{J}, N \in\{0.4,0.6,0.8,1,1.2,1.4,1.6,1.8,2\}, c \in$ $\{-3,-2,-1,0,1,2,3,4,5\}$, and $\mathbf{d}$ denotes the square-wave disturbance as stated before. Thus, the total inertia matrix is $\mathbf{J}=\mathbf{J}_{0}+c \Delta \mathbf{J}$. Moreover, the control gains and the initial value of the updating law Equation (41) are chosen to be the same for the cases.

Figures 2, 3, and 4 show the resulted sliding mode surface. It is observed that although all the states of the attitude tracking system reach the sliding surface (16) within 8.75 seconds even in the presence of different external disturbances and uncertain inertia parameters, each case is corresponding to the different finite-time $t^{*}$. Actually, due to different disturbance and uncertain inertia, $\mathbf{D}$ is different for each case. As a consequence, different $\widetilde{\mathbf{D}}\left(t_{0}\right)$ is obtained due to the same initial value of the updating law Equation (41) for different case. That leads to the different finite-time $t^{*}$ from (46), although the value of $K$ is all the same.

The attitude tracking error is illustrated in Figures 5, 6, and 7, respectively. The controller Equation (51) 


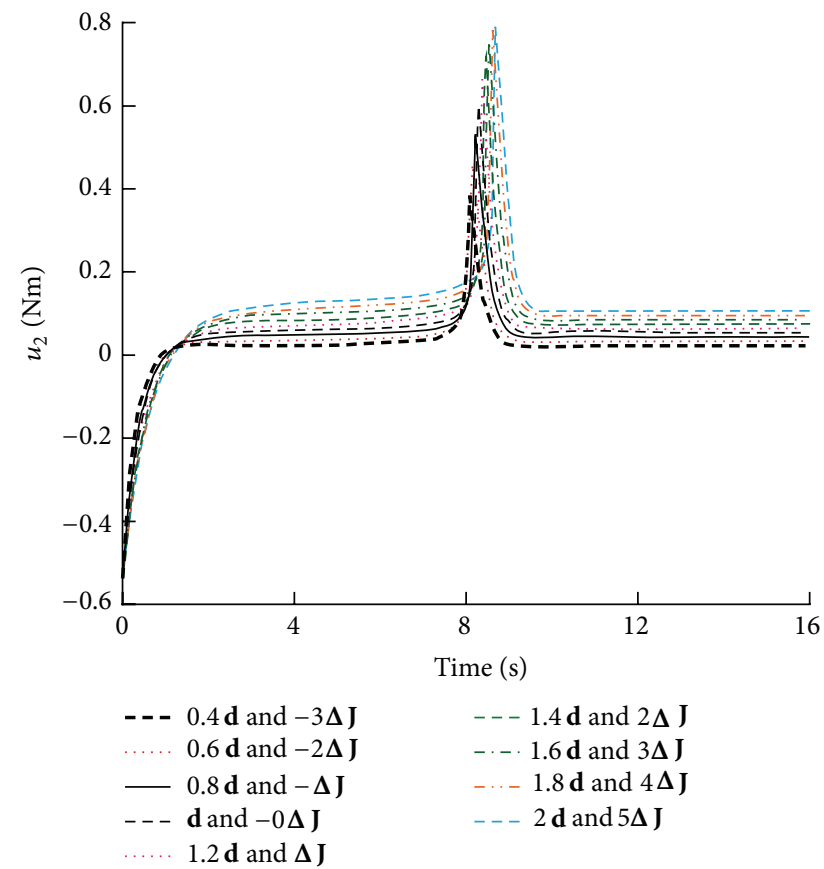

(a) The whole response

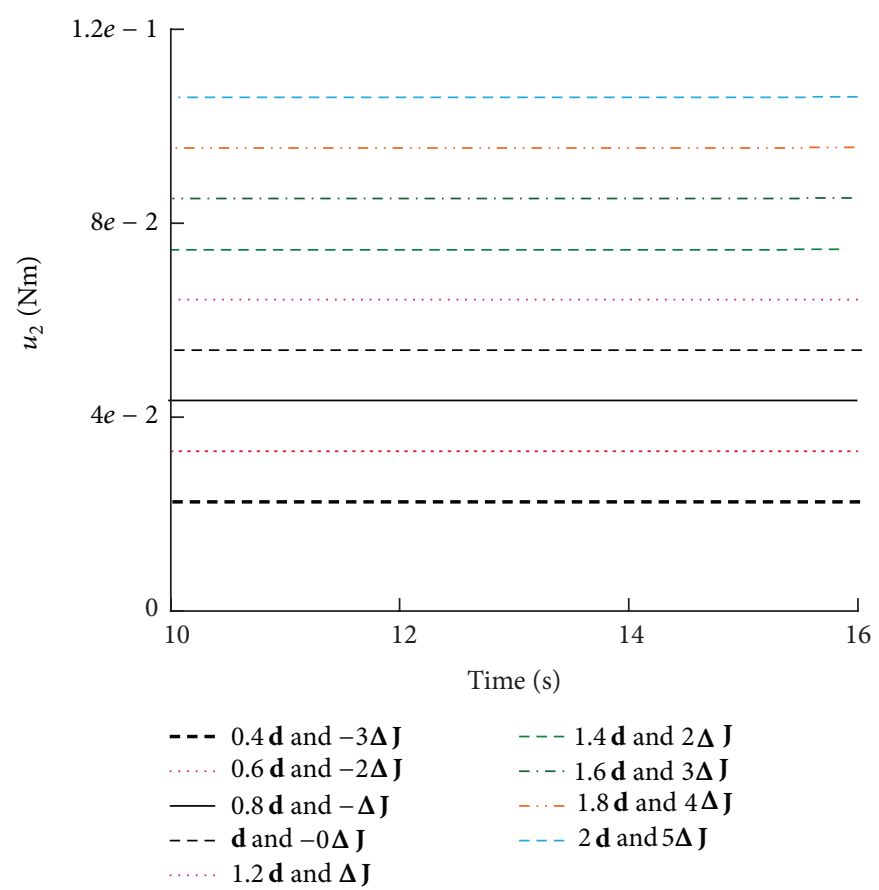

(b) The steady-state behavior

FIGURE 12: The control effort $u_{2}$ with different disturbance and uncertain inertia.

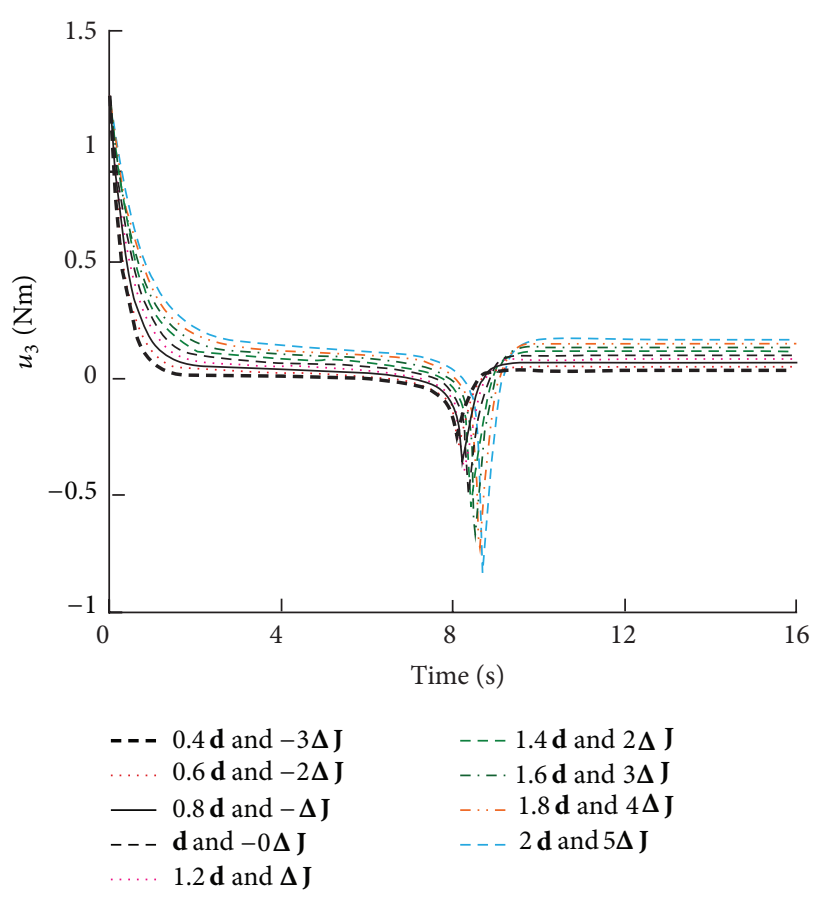

(a) The whole response

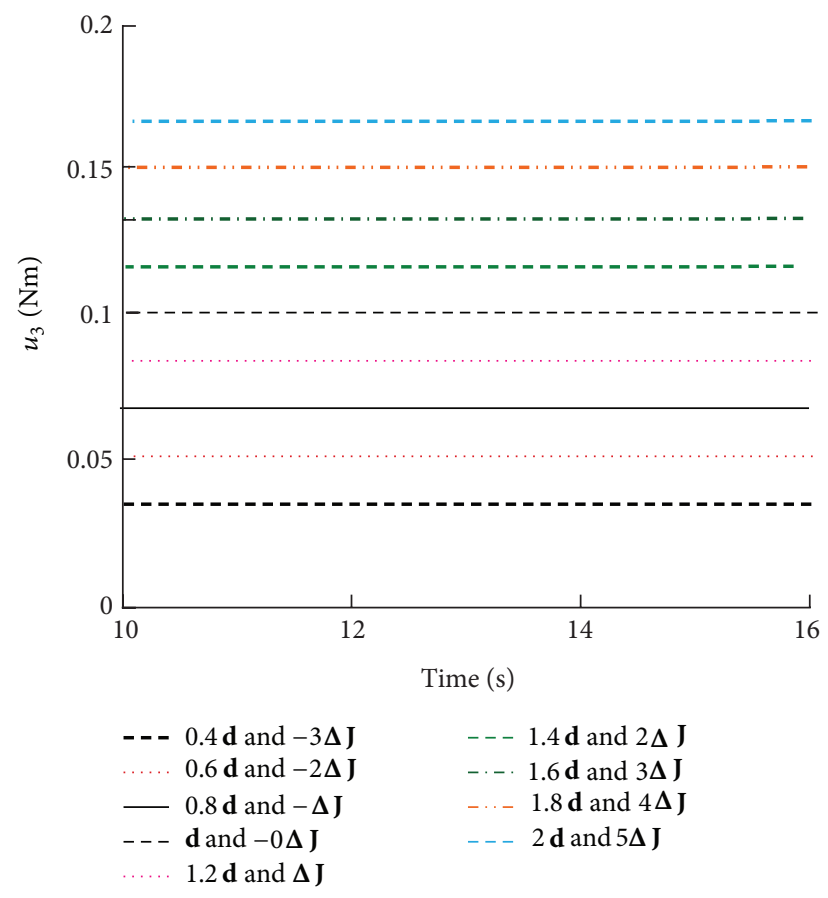

(b) The steady-state behavior

FIgURE 13: The control effort $u_{3}$ with different disturbance and uncertain inertia.

successfully accomplishes the attitude tracking maneuver. More specifically, as shown in Figures 5-7(a), the time response of the attitude tracking error is almost the same, although the satellite is under the effect of even nine types of disturbances and uncertain inertia. That is because this disturbance and uncertain inertia can be exactly estimated in finite time by using the adaptive law Equation (41) incorporated in the controller. Consequently, they can be compensated by the controller in finite time. On the other hand, it is seen in Figures 2-4(b) that the attitude tracking 
TABLE 1: The attitude control performance in the different cases.

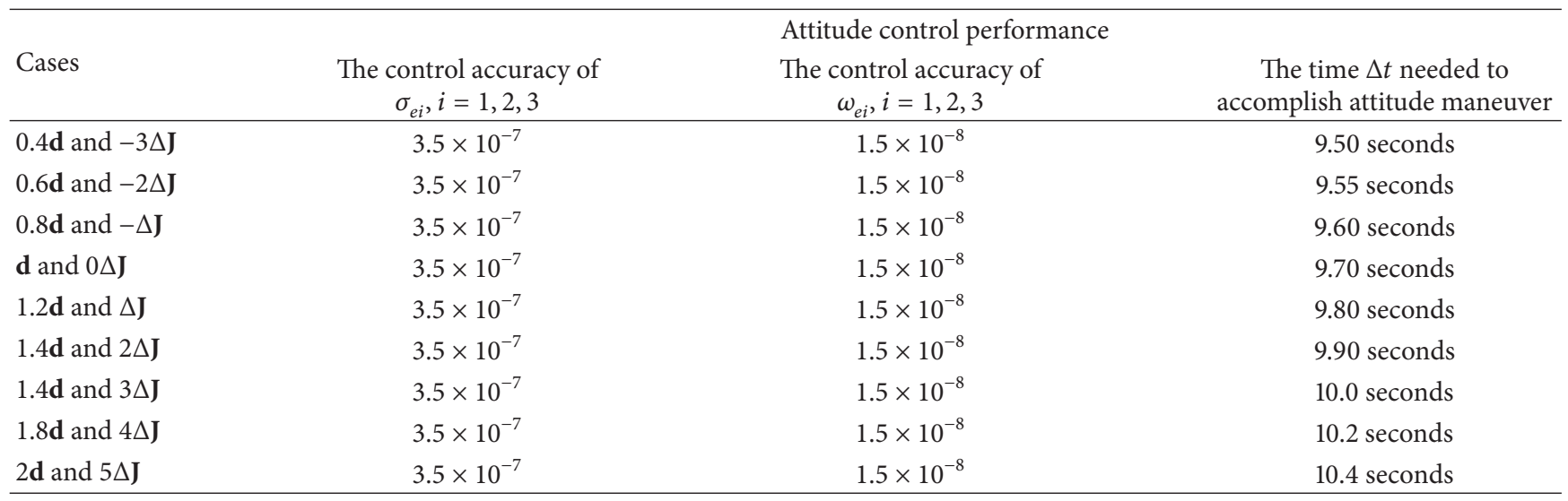

error is stabilized with high accuracy after 9.6 seconds. The angular velocity tracking error obtained from the controller Equation (51) is shown in Figures 8, 9, and 10, respectively. It is observed that the desired angular velocity is successfully followed almost within the same time, that is, 11.0 seconds. This control performance is guaranteed in case of nine types of uncertain disturbances and uncertain inertia parameters. Furthermore, it can be seen in Figures 4-9(b) that high attitude accuracy and attitude stability are obtained. The control $\mathbf{u}$ in the presence of difference disturbance and uncertain inertia are shown in Figures 11, 12, and 13, respectively.

Summarizing the above simulation results, the attitude control performance of different cases is listed in Table 1. It is noted that the proposed control approach is able to achieve attitude tracking maneuver even in the presence of different disturbances and uncertain inertia parameters. Great robustness to external disturbance and system uncertainty is guaranteed.

\section{Conclusions and Future Work}

An adaptive sliding mode control scheme was proposed to eliminate the effect of external disturbance and uncertain inertia parameters on a rigid satellite. With application of the proposed control, attitude tracking maneuver was successfully accomplished in finite time. The attitude tracking error and the velocity tracking error were asymptotically stabilized with finite-time convergence even in the presence of external disturbance and system uncertainties. Simulation results were presented to confirm the control performance of the controller. However, the problem of input saturation was not addressed. In practice, an input saturation occurrence of any actuator may lead to severe performance deterioration. As some of future works, extension of the approach to handle that problem should be carried out. Moreover, fault tolerant control should also be investigated to improve the reliability of the satellite.

\section{Conflict of Interests}

The authors declare that there is no conflict of interests regarding the publication of this paper.

\section{Acknowledgments}

This present work was supported partially by National Natural Science Foundation of China (Project no. 61304149) and Natural Science Foundation of Liaoning, China (Project no. 2013020044). The authors highly appreciate the above financial supports.

\section{References}

[1] J. Ahmed, V. T. Coppola, and D. S. Bernstein, "Adaptive asymptotic tracking of spacecraft attitude motion with inertia matrix identification," Journal of Guidance, Control, and Dynamics, vol. 21, no. 5, pp. 684-691, 1998.

[2] H. Yoon and B. N. Agrawal, "Adaptive control of uncertain hamiltonian multi-input multi-output systems: with application to spacecraft control," IEEE Transactions on Control Systems Technology, vol. 17, no. 4, pp. 900-906, 2009.

[3] D. Seo and M. R. Akella, "Separation property for the rigid-body attitude tracking control problem," Journal of Guidance, Control, and Dynamics, vol. 30, no. 6, pp. 1569-1576, 2007.

[4] D. Seo and M. R. Akella, "High-performance spacecraft adaptive attitude-tracking control through attracting-manifold design," Journal of Guidance, Control, and Dynamics, vol. 31, no. 4, pp. 884-891, 2008.

[5] C.-D. Yang and Y.-P. Sun, "Mixed $H_{2} / H_{\infty}$ state-feedback design for microsatellite attitude control," Control Engineering Practice, vol. 10, no. 9, pp. 951-970, 2002.

[6] Q. Zheng and F. Wu, "Nonlinear $H_{\infty}$ control designs with axisymmetric spacecraft control," Journal of Guidance, Control, and Dynamics, vol. 32, no. 3, pp. 850-859, 2009.

[7] R. Kristiansen, P. J. Nicklasson, and J. T. Gravdahl, "Satellite attitude control by quaternion-based backstepping," IEEE Transactions on Control Systems Technology, vol. 17, no. 1, pp. 227-232, 2009.

[8] X. Zhao, L. Zhang, P. Shi, and H. Karimi, "Novel stability criteria for TS fuzzy systems," 2013.

[9] X. Zhao, L. Zhang, P. Shi, and H. Karimi, "Robust control of continuous-time systems with state-dependent uncertainties and its application to electronic Circuits," 2013.

[10] C. K. Carrington and J. L. Junkins, "Optimal nonlinear feedack control for spacecraft attitude maneuvers," Journal of Guidance, Control, and Dynamics, vol. 9, no. 1, pp. 99-107, 1986. 
[11] W. Luo, Y.-C. Chu, and K.-V. Ling, "Inverse optimal adaptive control for attitude tracking of spacecraft," IEEE Transactions on Automatic Control, vol. 50, no. 11, pp. 1639-1654, 2005.

[12] R. J. Wallsgrove and M. R. Akella, "Globally stabilizing saturated attitude control in the presence of bounded unknown disturbances," Journal of Guidance, Control, and Dynamics, vol. 28, no. 5, pp. 957-963, 2005.

[13] L. Garcia, R. Farquhar, and T. Eastman, "New opportunities for a historic spacecraft," Space Weather, vol. 10, no. 8, 2012.

[14] S. Di Gennaro, "Adaptive robust tracking for flexible spacecraft in presence of disturbances," Journal of Optimization Theory and Applications, vol. 98, no. 3, pp. 545-568, 1998.

[15] T. Yamashita, N. Ogura, T. Kurii, and T. Hashimoto, "Improved satellite attitude control using a disturbance compensator," Acta Astronautica, vol. 55, no. 1, pp. 15-25, 2004.

[16] J. D. Bošković, S.-M. Li, and R. K. Mehra, "Robust tracking control design for spacecraft under control input saturation," Journal of Guidance, Control, and Dynamics, vol. 27, no. 4, pp. 627-633, 2004.

[17] Z.-X. Li and B.-L. Wang, "Robust attitude tracking control of spacecraft in the presence of disturbances," Journal of Guidance, Control, and Dynamics, vol. 30, no. 4, pp. 1156-1159, 2007.

[18] Z. Chen and J. Huang, "Attitude tracking and disturbance rejection of rigid spacecraft by adaptive control," IEEE Transactions on Automatic Control, vol. 54, no. 3, pp. 600-605, 2009.

[19] A. Sanyal, A. Fosbury, N. Chaturvedi, and D. S. Bernstein, "Inertia-free spacecraft attitude tracking with disturbance rejection and almost global stabilization," Journal of Guidance, Control, and Dynamics, vol. 32, no. 4, pp. 1167-1178, 2009.

[20] Q. Hu, B. Xiao, and M. I. Friswell, "Robust fault-tolerant control for spacecraft attitude stabilisation subject to input saturation," IET Control Theory and Applications, vol. 5, no. 2, pp. 271-282, 2011.

[21] C. Edwards and S. K. Spurgeon, Sliding Mode Control: Theory and Applications, Taylor \& Francis, 1998.

[22] W. Cai, X. H. Liao, and Y. D. Song, "Indirect robust adaptive fault-tolerant control for attitude tracking of spacecraft," Journal of Guidance, Control, and Dynamics, vol. 31, no. 5, pp. 1456-1463, 2008.

[23] T. A. W. Dwyer III and H. Sira-Ramirez, "Variable-structure control of spacecraft attitude maneuvers," Journal of Guidance, Control, and Dynamics, vol. 11, no. 3, pp. 262-270, 1988.

[24] Y.-P. Chen and S.-C. Lo, "Sliding-mode controller design for spacecraft attitude tracking maneuvers," IEEE Transactions on Aerospace and Electronic Systems, vol. 29, no. 4, pp. 1328-1333, 1993.

[25] C. Pukdeboon, A. S. I. Zinober, and M.-W. L. Thein, "Quasi-continuous higher order sliding-mode controllers for spacecraft-attitude-tracking maneuvers," IEEE Transactions on Industrial Electronics, vol. 57, no. 4, pp. 1436-1444, 2010.

[26] S. N. Wu, X. Y. Sun, Z. W. Sun, and X. D. Wu, "Slidingmode control for staring-mode spacecraft using a disturbance observer," Proceedings of the Institution of Mechanical Engineers, Part G: Journal of Aerospace Engineering, vol. 224, no. 2, pp. 215224, 2010.

[27] Z. Zhu, Y. Xia, and M. Fu, "Adaptive sliding mode control for attitude stabilization with actuator saturation," IEEE Transactions on Industrial Electronics, vol. 58, no. 10, pp. 4898-4907, 2011.

[28] Y. Xia, Z. Zhu, M. Fu, and S. Wang, "Attitude tracking of rigid spacecraft with bounded disturbances," IEEE Transactions on Industrial Electronics, vol. 58, no. 2, pp. 647-659, 2011.
[29] Q. Hu and B. Xiao, "Fault-tolerant sliding mode attitude control for flexible spacecraft under loss of actuator effectiveness," Nonlinear Dynamics, vol. 64, no. 1-2, pp. 13-23, 2011.

[30] E. Jin and Z. Sun, "Robust controllers design with finite time convergence for rigid spacecraft attitude tracking control," Aerospace Science and Technology, vol. 12, no. 4, pp. 324-330, 2008.

[31] Y. Cao, W. Ren, and Z. Meng, "Decentralized finite-time sliding mode estimators and their applications in decentralized finitetime formation tracking," Systems and Control Letters, vol. 59, no. 9, pp. 522-529, 2010.

[32] H. Du and S. Li, "Finite-time attitude stabilization for a spacecraft using homogeneous method," Journal of Guidance, Control, and Dynamics, vol. 35, pp. 740-748, 2012.

[33] S. Yu, X. Yu, B. Shirinzadeh, and Z. Man, "Continuous finitetime control for robotic manipulators with terminal sliding mode," Automatica, vol. 41, no. 11, pp. 1957-1964, 2005.

[34] H. Ando, T. Imamura, A. Nabatov et al., "Dual-spacecraft radio occultation measurement of the electron density near the lunar surface by the SELENE mission," Journal of Geophysical Research-Space Physics, vol. 117, no. A8, 2012.

[35] F.-K. Yeh, "Sliding-mode adaptive attitude controller design for spacecrafts with thrusters," IET Control Theory and Applications, vol. 4, no. 7, pp. 1254-1264, 2010.

[36] S. Ding and S. Li, "Stabilization of the attitude of a rigid spacecraft with external disturbances using finite-time control techniques," Aerospace Science and Technology, vol. 13, no. 4-5, pp. 256-265, 2009.

[37] Z. Zhu, Y. Xia, and M. Fu, "Attitude stabilization of rigid spacecraft with finite-time convergence," International Journal of Robust and Nonlinear Control, vol. 21, no. 6, pp. 686-702, 2011.

[38] G. Godard and K. D. Kumar, "Robust attitude stabilization of spacecraft subject to actuator failures," Acta Astronautica, vol. 68, no. 7-8, pp. 1242-1259, 2011.

[39] A.-M. Zou, K. D. Kumar, Z.-G. Hou, and X. Liu, "Finite-time attitude tracking control for spacecraft using terminal sliding mode and chebyshev neural network," IEEE Transactions on Systems, Man, and Cybernetics, Part B, vol. 41, no. 4, pp. 950963, 2011.

[40] G. Godard and K. D. Kumar, "Fault tolerant reconfigurable satellite formations using adaptive variable structure techniques," Journal of Guidance, Control, and Dynamics, vol. 33, no. 3, pp. 969-984, 2010.

[41] D. Shin, G. Moon, and Y. Kim, "Design of reconfigurable flight control system using adaptive sliding mode control: actuator fault," Proceedings of the Institution of Mechanical Engineers, Part G: Journal of Aerospace Engineering, vol. 219, no. 4, pp. 321-328, 2005.

[42] J. L. Crassidis and F. L. Markley, "Sliding mode control using modified Rodrigues parameters," Journal of Guidance, Control, and Dynamics, vol. 19, no. 6, pp. 1381-1383, 1996.

[43] H. Wang, Z.-Z. Han, Q.-Y. Xie, and W. Zhang, "Finite-time chaos control via nonsingular terminal sliding mode control," Communications in Nonlinear Science and Numerical Simulation, vol. 14, no. 6, pp. 2728-2733, 2009.

[44] M. J. Sidi, Spacecraft Dynamics and Control, Cambridge University Press, Cambridge, UK, 1997. 


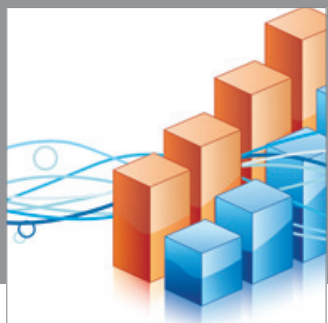

Advances in

Operations Research

mansans

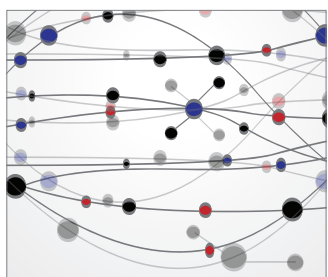

The Scientific World Journal
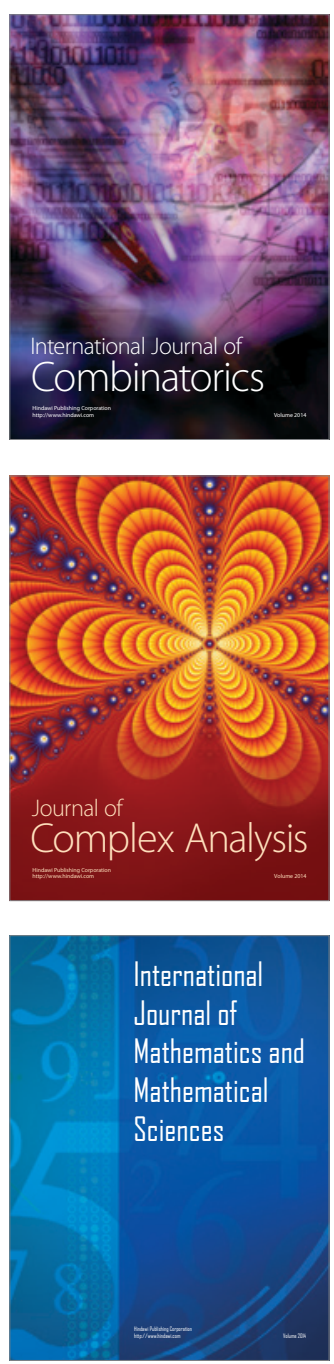
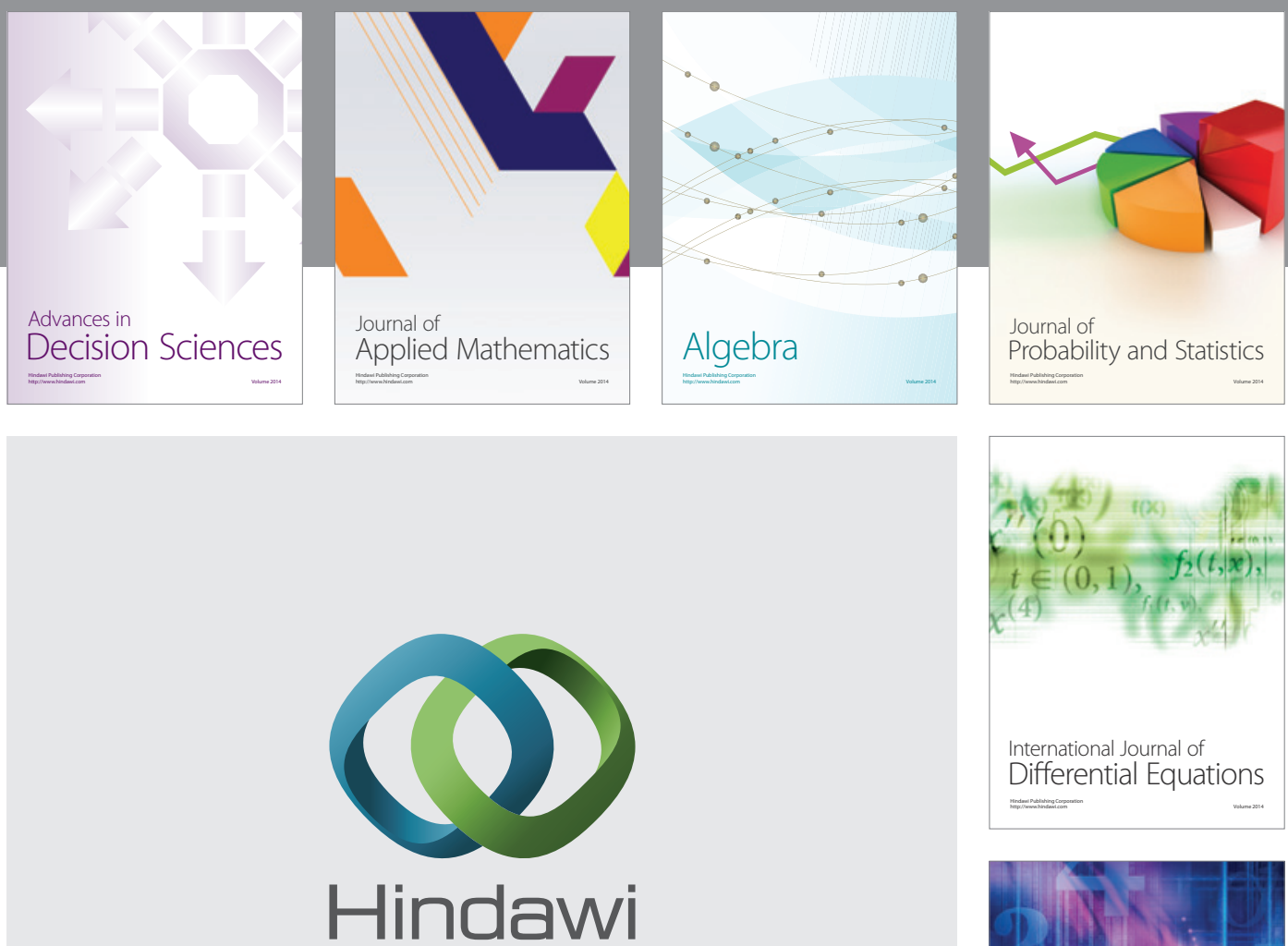

Submit your manuscripts at http://www.hindawi.com
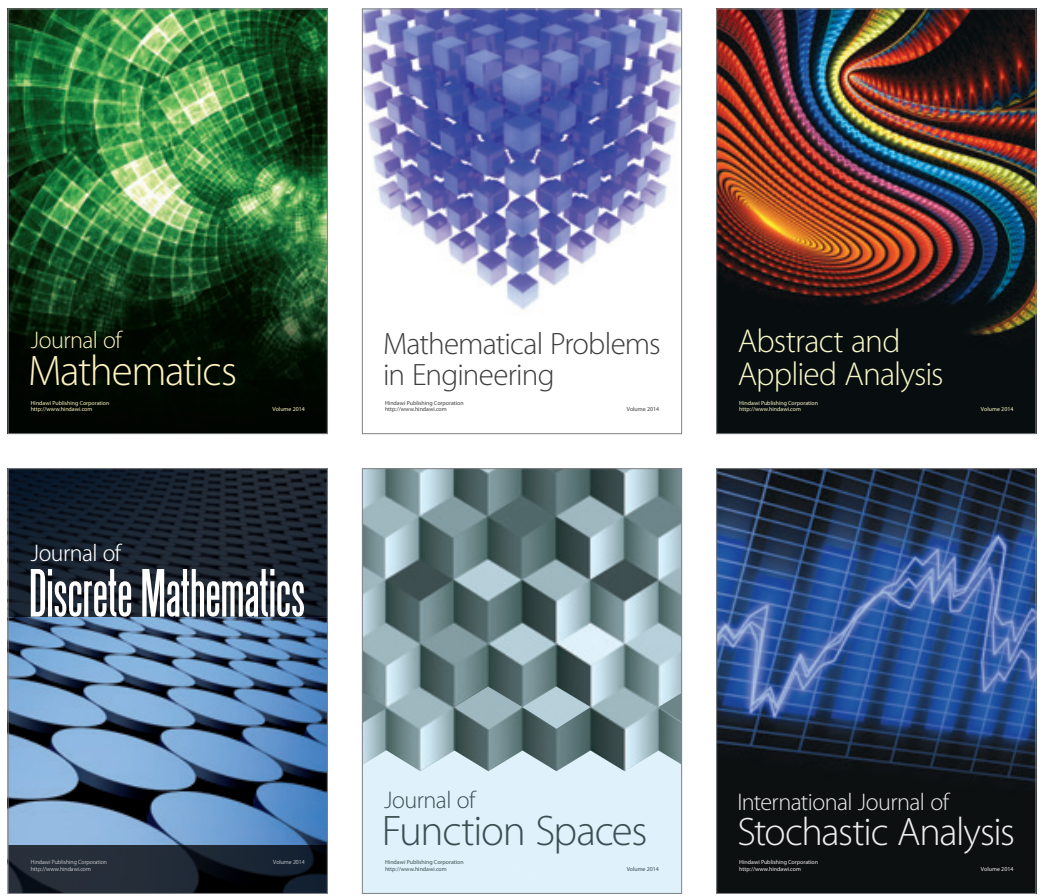

Journal of

Function Spaces

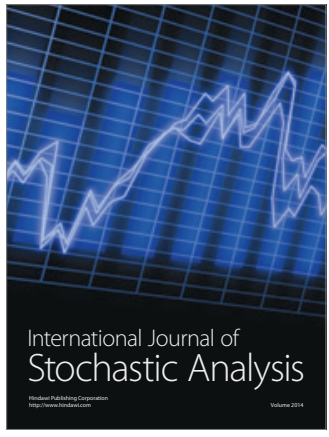

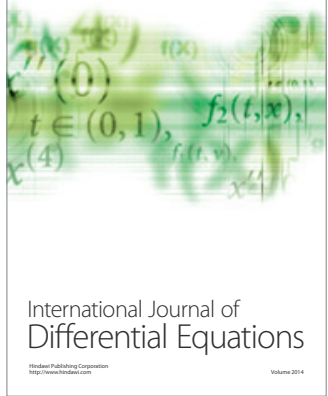
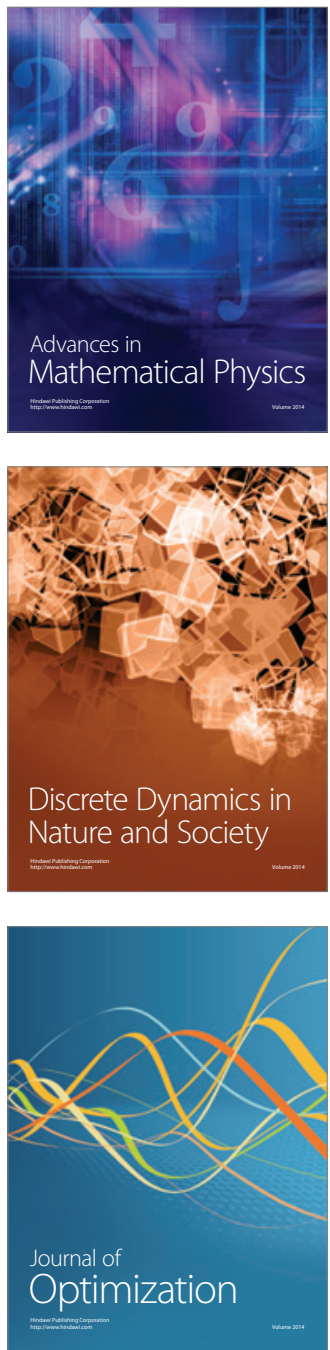